\title{
Números de Reidemeister Relativos
}

\author{
Aldemir José da Silva Pinto
}

\author{
TESE APRESENTADA AO \\ INSTITUTO DE MATEMÁTICA E ESTATÍSTICA \\ DA UNIVERSIDADE DE SÃO PAULO \\ PARA OBTENÇÃO DO GRAU DE \\ DOUTOR EM MATEMÁTICA
}

Área de Concentração: Topologia Algébrica Orientadora: Profa. Dra. Fernanda Soares Pinto Cardona
Apoio Financeiro: Departamento de Matemática /UFPR e
CAPES

- São Paulo, Julho de 2006 - 


\section{Números de Reidemeister Relativos}

Este exemplar corresponde à redação final da tese corrigida e defendida por Aldemir José da Silva Pinto e aprovada pela Comissão Julgadora.

São Paulo, Julho de 2006.

Banca Examinadora:

Profa. Dra. Fernanda Soares Pinto Cardona (orientadora) - IME/USP

Profa. Dra. Lucília Daruiz Borsari - IME/USP

Prof. Dr. Peter Wong - BATES COLLEGE/USA

Prof. Dr. Pedro Luiz Queiroz Pergher - UFSCar

Profa. Dra. Alice Kimie Miwa Libardi - UNESP/Rio Claro 
Dedico a minha mãe Carmem Silva Pinto (in memoriam) e a Mauro José da Silva Pinto (in memoriam). 


\section{Agradecimentos}

Gostaria de expressar a minha profunda gratidão à Profa. Dra. Fernanda Soares Pinto Cardona, pela orientação e apoio desde o primeiro ano do Doutorado, e aos professores do grupo de Topologia Algébrica do IME, Profa. Dra. Lucília Daruiz Borsari e Prof. Dr. Daciberg Lima Gonçalves.

Agradeço também a Profa. Dra. Célia Contin Góes (IME/USP), a Profa. Dra. Ofélia Teresa Alas (IME/USP), ao Prof. Dr. Alfredo Jorge Aragona (IME/USP), ao Prof. Dr. Luiz Pedro Orosz (UFES), ao Prof. Standard Silva (UFES), ao Prof. Dr. Pedro Donizeti (UFPR), ao Prof. Antônio Carlos (UFPR) e a Profa. Maria Tereza Spínola de Miranda (Guarapari/ES), que sempre me incentivaram nos estudos matemáticos, e a todos os meus amigos visíveis e invisíveis. 


\section{Resumo}

Neste trabalho definimos um número de Reidemeister, $\mathrm{R}\left(f ; A_{1} \cup A_{2}\right)$, para as auto-aplicações das tríades. Esse número satisfaz as propriedades esperadas do número de Reidemeister, $\mathrm{R}(f)$, na teoria do ponto fixo. Para tanto, redefinimos outros números de Reidemeister, obtendo uma facilidade maior no cálculo dos mesmos e novas propriedades. Além disso, apresentamos caracterizações algébricas para o número de Reidemeister da tríade. 


\begin{abstract}
In this work we define a Reidemeister number, $\mathrm{R}\left(f ; A_{1} \cup A_{2}\right)$, for selfmaps of triads. This number satisfies the expected properties of the Reidemeister number, $\mathrm{R}(f)$, in fixed point theory. In order to do that, we redefine other Reidemeister numbers, thus obtaining easier calculations and new properties. Also, we present algebraic caracterizations for the Reidemeister number of the triade.
\end{abstract}




\section{Índice}

Introdução iv

1 Conceitos Preliminares 1

2 Teorias de Reidemeister do Complemento e Relativa 19

3 O Número de Reidemeister da Tríade 38

3.2 Propriedades do número de Reidemeister da tríade . . . . . . . . 42

3.3 Exemplos . . . . . . . . . . . . . . . . . . . . . . . . 49

4 Caracterização Algébrica do Número de Reidemeister da Tríade 56

4.1 Formulação Algébrica por $\varphi$-conjugação . . . . . . . . . . . . 57

4.2 Formulação Algébrica para Espaços de Jiang . . . . . . . . . . . . 63 


\section{Chapter 1}

\section{Introdução}

Seja $X$ um poliedro compacto, conexo e uma aplicação $f: X \longrightarrow X$. Um problema central na teoria de ponto fixo é encontrar o número mínimo de pontos fixos, $\operatorname{MF}[f]$, na classe de homotopia de $f$. A teoria de Nielsen, que se iniciou por volta de 1927 com o artigo de J. Nielsen, Untersuchungen zur Topologie der gescholossenen zweiseitigen Flächen, é um dos possíveis enfoques para responder tal questão. Ela foi desenvolvida nos trabalhos de R. Brown [1], B. Jiang [7], H. Schirmer [11] e X. Zhao [15], entre outros. O número de Reidemeister, $\mathrm{R}(f)$, que foi introduzido em 1936 por K. Reidemeister no artigo Automorphismen von Homotopiekettenringen, é um limitante superior para o número de Nielsen e, sob algumas condições, por exemplo quando $X$ é espaço de Jiang, temos $\mathrm{R}(f)=\mathrm{N}(f)$. Com a abordagem utilizada por Jiang [7], o cálculo de $\mathrm{R}(f)$ é mais fácil que o de $\mathrm{N}(f)$, o que o torna uma ferramenta útil no problema de determinação do número 
$\operatorname{MF}[f]$.

Em 1986, Helga Schirmer em [11] introduziu a noção do número de Nielsen relativo, $\mathrm{N}(f ; X, A)$, para aplicações $f:(X, A) \longrightarrow(X, A)$, onde $X$ e $A \subset X$ são poliedros compactos e $X$ é conexo, uma vez que o número de Nielsen de $f$, não fornece informações suficientes sobre as homotopias de um par de espaços, não sendo assim um bom limitante para $\operatorname{MF}[f ; X, A]$.

Em 1988, X. Zhao, em [15], definiu o número de Nielsen relativo para o complemento, $\mathrm{N}(f ; X-A)$, a fim de descrever de forma mais precisa o comportamento do número mínimo de pontos fixos das autoaplicações de pares de espaços no espaço complementar, $M F[f ; X-A]$, pois nem o número de Nielsen, $\mathrm{N}(f)$ nem o número de Nielsen relativo, $\mathrm{N}(f ; X, A)$, o fazem. Impondo algumas condições nos espaços envolvidos, ele demonstrou que o número mínimo de pontos fixos do complemento de uma aplicação é atingido pelo número de Nielsen do complemento.

H. Schirmer estudou o problema do número mínimo de pontos fixos para as tríades $\left(X, A_{1}, A_{2}\right)$, onde $X$ é um espaço compacto e conexo, $X=A_{1} \cup A_{2}$ e $A_{1}, A_{2}$ são subespaços de $X$. Novamente verificou-se que os números de Nielsen definidos até então não eram bons limitantes inferiores para $\operatorname{MF}\left[f ; A_{1} \cup A_{2}\right]$. Em 1993, no 
artigo [12], Schirmer definiu o número de Nielsen, $\mathrm{N}\left(f ; A_{1} \cup A_{2}\right)$, para aplicações da tríade. Além de ter as propriedades usuais de um número de Nielsen, ela demonstrou que $\mathrm{N}\left(f ; A_{1} \cup A_{2}\right)$ é um bom limitante inferior para $\mathrm{MF}\left[f ; A_{1} \cup A_{2}\right]$, que atinge o mínimo quando os espaços em questão satisfazem algumas hipóteses.

Em [2] foram apresentados números de Reidemeister relativo e o do complemento, respectivamente $R(f ; X, A)$ e $R(f ; X-A)$, que satisfazem quase todas as propriedades desejadas, análogas às do número de Reidemeister clássico, mas que não são invariantes homotópicos. Resolveu-se esse problema definindo $R^{*}(f ; X, A)$ e $R^{*}(f ; X-A)$, os respectivos valores mínimos assumidos por $R(f ; X, A)$ e $R(f ; X-A)$, na classe das aplicações homotópicas à $f$.

Neste trabalho melhoramos os resultados obtidos em [2], definindo de maneira diferente os números de Reidemeister relativo e do complemento que satisfazem propriedades análogas às de $\mathrm{R}(f)$, inclusive a da invariância homotópica. Além disso, definimos o número de Reidemeister, $\mathrm{R}\left(f ; A_{1} \cup A_{2}\right)$, no contexto das autoaplicações da tríade.

Com o intuito de tornar a leitura deste trabalho mais simples, no Capítulo 1 apresentamos de maneira sucinta os conceitos básicos utilizados, cujas demons- 
trações completas podem ser encontradas em [7] e [8]. Também incluímos diversos exemplos, ilustrando as possíveis situações decorrentes da aplicação de [15, Proposition 2.1].

Na primeira parte do Capítulo 2 apresentamos um resumo dos resultados obtidos por X. Zhao [15] para o número de Nielsen do complemento. Em seguida, fazemos uma nova definição do número de Reidemeister do complemento obtendo novos resultados. Na segunda parte fazemos o mesmo para número de Reidemeister relativo.

No Capítulo 3 definimos o número de Reidemeister da tríade, $\mathrm{R}\left(f ; A_{1} \cup A_{2}\right)$ e demonstramos que ele é um limitante superior para o número de Nielsen da tríade. Além disso, provamos sua invariância homotópica e que ele possui a propriedade dos espaços de Jiang. Observamos também que ele pode ser encarado como uma generalização do número de Reidemeister clássico e do número de Reidemeister relativo, e apresentamos alguns exemplos.

No Capítulo 4 usamos os conceitos desenvolvidos nos artigos [3] e [15], para reestabelecer caracterizações algébricas para os números de Reidemeister do complemento e relativo (segundo as novas definições) e obtivemos caracterizações 


\section{Capítulo 1}

\section{Conceitos Preliminares}

Neste capítulo procuramos agrupar as definições e resultados básicos para uma maior compreensão e desenvolvimento da teoria. Como o resultado ([15], Proposição 2.1) é fundamental para o trabalho, no final deste capítulo apresentamos exemplos das diversas situações possíveis, decorrentes da aplicação do mesmo.

Definição 1.1. Um espaço de recobrimento de $X$ é uma terna $E \stackrel{p}{\rightarrow} X$ se todo $x \in X$ tem uma vizinhança aberta $U$ tal que $p^{-1}(U)$ é união disjunta de abertos $S_{i}$ de $E$, cada um dos quais são homeomorfos a $U$ pela aplicação $p$. Um espaço de recobrimento $\tilde{X} \stackrel{p}{\rightarrow} X$ é dito universal se $\tilde{X}$ é simplesmente conexo.

No que segue estaremos trabalhando com o recobrimento universal.

Definição 1.2. Seja $f: X \rightarrow X$ contínua, onde $X$ é um poliedro compacto $e$ conexo. A aplicação $\tilde{f}: \widetilde{X} \rightarrow \tilde{X}$ é um levantamento de $f$ se $p \circ \tilde{f}=f \circ p$. 
Dois levantamentos $\tilde{f}$ e $\tilde{f}^{\prime}$ de $f: X \rightarrow X$, onde $X$ é um poliedro compacto $e$ conexo, são ditos conjugados se, para $p: \widetilde{X} \rightarrow X$ aplicação de revestimento, existe $\gamma \in \mathcal{D}(\tilde{X})=\{\gamma: \tilde{X} \rightarrow \tilde{X} \mid p \circ \gamma=p\}$ tal que $\tilde{f}^{\prime}=\gamma \circ \tilde{f} \circ \gamma^{-1}$.

As classes de levantamento são as classes de equivalência dos levantamentos por conjugação, denotadas por

$$
[\tilde{f}]=\left\{\gamma \circ \tilde{f} \circ \gamma^{-1} \mid \gamma \in \mathcal{D}(\tilde{X})\right\}
$$

Como o conjunto dos pontos fixos de $f$ e de $\tilde{f}$ têm as seguintes propriedades:

(i) $\operatorname{Fix}(f)=\bigcup_{\tilde{f}} p F i x(\tilde{f})$

(ii) $p F i x(\tilde{f})=p F i x \tilde{f}^{\prime}$, se $[\tilde{f}]=\left[\tilde{f}^{\prime}\right]$

(iii) $p F i x(\tilde{f}) \cap p F i x \tilde{f}^{\prime}=\emptyset$, se $[\tilde{f}] \neq\left[\tilde{f}^{\prime}\right]$ (para maiores detalhes, veja [2] e [8]), podemos fazer a seguinte definição:

Definição 1.3. O subconjunto $p F i x(\tilde{f})$ de $F i x(f)$ denomina-se a classe de ponto fixo de $f$ determinada pela classe de levantamento $[\tilde{f}]$.

Além disso, podemos provar que:

Teorema 1.1. O conjunto Fix $(f)$ se escreve como uma reunião disjunta de classes de pontos fixos. 
Demonstração: Imediata.

Boju Jiang em [7] considera a classe de ponto fixo de $f$ determinada pela classe de levantamento $[\tilde{f}]$ como um par $(p F i x(\tilde{f}),[\tilde{f}])$, denominado a classe de ponto fixo rotulada. Nesse sentido temos uma correspondência entre as classes de levantamento de $f$ e as classes de pontos fixos. Isto nos leva à definição:

Definição 1.4. O número de classes de levantamento de $f$ (bem como o número de classes de pontos fixos, vazias ou não) denomina-se número de Reidemeister de $f$, denotado por $R(f)$.

Observe que $R(f)$ é um número inteiro positivo ou infinito.

Esta definição essencialmente diz que: Dois pontos fixos de $f$ estão na mesma classe se, e somente se, existe um levantamento $\tilde{f}$ de $f$ que tenha pontos fixos em suas pré-imagens.

O número $R(f)$ é um invariante homotópico, pois temos que:

Teorema 1.2. Sejam as aplicações $f_{0}, f_{1}: X \rightarrow X$. Se existe uma homotopia $H: f_{0} \simeq f_{1}$, então existe uma correspondência entre as classes de levantamento de $f_{0}$ e as de $f_{1}$. 
Demonstração: [7]

Além disso, o número de Reidemeister tem as seguintes propriedades:

Propriedade 1.1 (Propriedade Comutativa). Sejam $X$ e $Y$ poliedros compactos e conexos e $f: X \rightarrow Y, g: Y \rightarrow X$ aplicações. Então $R(f \circ g)=R(g \circ f)$.

Demonstração: [2]

Propriedade 1.2 (Propriedade do Produto). Sejam $X$ e $Y$ poliedros compactos e conexos, $f: X \rightarrow X, g: Y \rightarrow Y$ aplicações e $f \times g: X \times Y \rightarrow X \times Y$ definida por $(f \times g)(x, y)=(f(x), g(y))$. Então $R(f \times g)=R(f) R(g)$.

Demonstração: [2]

Propriedade 1.3. Se $X$ e $Y$ são poliedros compactos e conexos, $f: X \rightarrow X$ e $g: Y \rightarrow Y$ têm o mesmo tipo de homotopia, então $R(f)=R(g)$.

Demonstração: [2]

O conceito de índice de ponto fixo é indispensável na teoria de pontos fixos. 
Basicamente definimos o índice de um ponto fixo isolado para auto-aplicações do $\mathbb{R}^{n}$ (usando o grau de uma aplicação adequada) e, sabendo que todo poliedro pode ser mergulhado em algum $\mathbb{R}^{n}$, estendemos a definição de índice para as autoaplicações de poliedros compactos. (Para maiores detalhes veja, por exemplo, [5] e [6].)

Seja uma aplicação $f: X \rightarrow X$, onde $X$ é um poliedro compacto e conexo. Seja $\mathbb{F}$ uma classe de pontos fixos de $f$. Sabemos que $\mathbb{F}$ é um conjunto de pontos fixos isolados. Assim, o índice de $f$ em $\mathbb{F}$, denotado por $\operatorname{ind}(f, \mathbb{F})$, é um número bem definido.

Definição 1.5. Dizemos que $\mathbb{F}$ é uma classe de pontos fixos essencial de $f$ se ind $(f, \mathbb{F}) \neq 0$. Se ind $(f, \mathbb{F})=0$, dizemos que $\mathbb{F}$ é uma classe de pontos fixos inessencial.

Analogamente definimos:

(i) A classe de levantamento $[\tilde{f}]$ é essencial se $p F i x(\tilde{f}) \neq \emptyset$ e é essencial;

(ii) A classe de levantamento $[\tilde{f}]$ é inessencial se $p F i x(\tilde{f}) \neq \emptyset$ e é inessencial, ou $p \operatorname{Fix}(\tilde{f})=\emptyset$. 
Definição 1.6. $O$ número de Nielsen de $f$, denotado por $N(f)$, é o número de classes de pontos fixos essenciais de $f$, isto é,

$$
N(f)=\#\{p F i x(\tilde{f}) \mid \operatorname{ind}(f, p F i x(\tilde{f})) \neq 0\}
$$

O número de Nielsen tem as seguintes propriedades que derivam diretamente das propriedades do índice de um ponto fixo isolado ou decorrem da definição:

Teorema 1.3. Seja $f: X \rightarrow X$ com $X$ poliedro compacto e conexo. Então:

(i) $N(f) \leq R(f)$;

(ii) Cada classe de pontos fixos essenciais é um conjunto não vazio;

(iii) $0 \leq N(f)<\infty$;

(iv) $N(f) \leq \# F i x(f)$;

(v) Se $f_{0}, f_{1}: X \rightarrow X$ são homotópicas, então $N\left(f_{0}\right)=N\left(f_{1}\right)$;

(vi) $N(f) \leq \min \{\# F i x(g) \mid g \simeq f\}$;

(vii) Seja $\mathbb{F}$ uma classe não vazia de pontos fixos de $g \circ f$. Então $f(\mathbb{F})$ é uma classe de pontos fixos de $f \circ g$ e ind $(g \circ f, \mathbb{F})=\operatorname{ind}(f \circ g, f(\mathbb{F}))$. Assim, $N(g \circ f)=N(f \circ g)$ 
(viii) Sejam $X$ e $Y$ poliedros compactos e conexos. Se $f: X \rightarrow X$ e $g: Y \rightarrow Y$ sẫo aplicações com o mesmo tipo de homotopia, então $N(f)=N(g)$.

\section{Demonstração: [8]}

Seja $f: X \rightarrow X$ aplicação onde $X$ é um poliedro compacto e conexo.

Definição 1.7. O subgrupo $J\left(f, x_{0}\right) \subset \pi_{1}\left(X, f\left(x_{0}\right)\right)$ denomina-se Grupo de Jiang de $f$ e é definido por

$$
J\left(f, x_{0}\right)=\left\{\alpha \in \pi_{1}\left(X, f\left(x_{0}\right)\right) \mid \exists H=\left\{h_{t}\right\}: f \simeq f, \operatorname{com}\left\langle H\left(x_{0}\right)\right\rangle=\alpha\right\}
$$

Usamos a notação $J(X)=J\left(i d_{X}, x_{0}\right) \subset \pi_{1}\left(X, x_{0}\right)$.

Definição 1.8. Um espaço $X$ é de Jiang se $J(X)=\pi_{1}\left(X, x_{0}\right)$.

Como exemplos de espaços de Jiang temos os espaços simplesmente conexos, os espaços generalizados de Lens $L\left(m ; q_{1}, \ldots, q_{n}\right)$, os $H$-espaços e observamos que produtos cartesianos de espaços de Jiang também é espaço de Jiang.

Teorema 1.4 (Propriedade dos Espaços de Jiang). Seja $X$ um poliedro compacto e conexo. Se X é um espaço de Jiang, entẫo quaisquer duas classes de 
pontos fixos de $f$ têm o mesmo índice. Além disso, se $L(f)=0$, então $N(f)=0$; se $L(f) \neq 0$, então $N(f)=R(f)=\# \operatorname{Coker}\left(1-f_{1_{*}}\right)$.

\section{Demonstração: [8]}

Em [15], Xuezhi Zhao definiu a aplicação $i_{k, F P C}$ entre classes de levantamentos (ou de pontos fixos) para uma aplicação do par $f:(X, A) \rightarrow(X, A)$.

Seja $f:(X, A) \rightarrow(X, A)$ aplicação de um par de poliedros compactos ( $A$ não necessariamente conexo) e $\hat{A}=\bigcup_{k=1}^{n} A_{k}$ a reunião de todas as componentes de $A$ que satisfazem $f\left(A_{k}\right) \subseteq A_{k}$, sendo $f_{A}: A \rightarrow A$ a restrição de $f$ ao subespaço $A$ e $f_{k}: A_{k} \rightarrow A_{k}$ a restrição de $f$ ao subespaço $A_{k}$.

O espaço $X$ e as componentes de $\hat{A}$ possuem coberturas universais dadas por

$$
p: \widetilde{X} \rightarrow X \quad \text { e } \quad p_{k}: \widetilde{A}_{k} \rightarrow A_{k}, \quad k=1,2, \ldots, n .
$$

Como $A \subset X$ não é necessariamente conexo, estendemos o conceito de recobrimento universal da maneira usual: $\tilde{A}=\bigcup \tilde{A}_{i}$, onde $\tilde{A}$ é o recobrimento universal da componente $A_{i}$ de $A$.

Consideremos o seguinte morfismo $i_{k}$ de $f_{k}$ para $f$ (onde $i_{k}: A_{k} \rightarrow X$ é a 
inclusão):

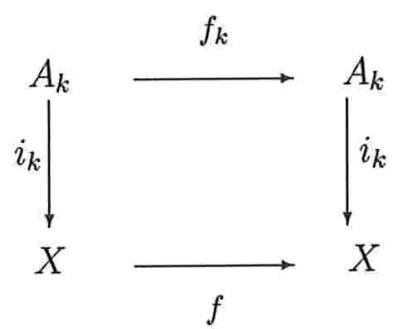

Para cada $k=1,2, \ldots, n$ consideremos um levantamento $\tilde{i}_{k}$ de $i_{k}$. Temos então que: Dados $\tilde{f}_{k}, \tilde{i}_{k}$ existe um levantamento $\tilde{f}$ de $f$ tal que o diagrama

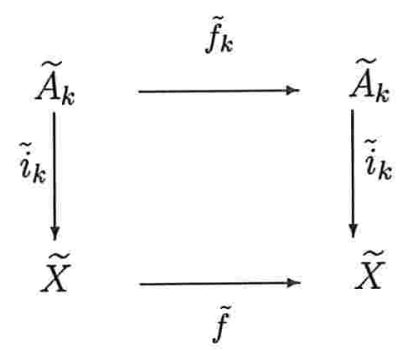

comuta, isto é, $\tilde{i}_{k} \circ \tilde{f}_{k}=\tilde{f} \circ \tilde{i}_{k}$.

Assim, determinamos uma correspondência

$$
\begin{aligned}
\tilde{i}_{k}: \operatorname{Lift}_{\tilde{f}_{k}}\left(f_{k}\right) & \longrightarrow \operatorname{Lift}(f) \\
\tilde{f}_{k} & \longmapsto
\end{aligned}
$$

Esta correspondência independe do levantamento $\tilde{i}_{k}$ de $i_{k}$, e é determinada apenas por $i_{k}$, sendo denotada por

$$
i_{k, F P C}: F P C\left(f_{k}\right) \rightarrow F P C(f)
$$

onde $F P C(f)$ é a classe de ponto fixo rotulada de $f$.

Temos então: 
Proposição 1.1. Cada classe de ponto fixo de $f_{k}: A_{k} \rightarrow A_{k}$ pertence a alguma classe de ponto fixo de $f$. Além disso, quando $p_{k} F i x\left(\tilde{f}_{k}\right)$ é não vazia temos que

$$
p_{k} \operatorname{Fix}\left(\tilde{f}_{k}\right) \in p F i x(\tilde{f}) \Longleftrightarrow i_{k, F P C}\left[\tilde{f}_{k}\right]=[\tilde{f}]
$$

(Mesmo quando $p_{k} F i x\left(\tilde{f}_{k}\right)=\emptyset$ teremos uma correspondência entre as classes de levantamento).

Demonstração: [15].

A aplicação $i_{k, F P C}$ nos sugere que existem possibilidades de relações entre as classes de levantamento essenciais e inessenciais. Podemos ter classes de levantamento essenciais contidas em inessenciais e vice-versa, e todos os casos possíveis, como nos mostram os exemplos a seguir:

Exemplo 1.1. Classe de levantamento inessencial de $f$ que contém classe de levantamento inessencial de $f_{A}$ :

Sejam $X=S^{1} \times[0,1], A=S^{1} \times\{0\}$, e seja

$$
\begin{aligned}
f:(X, A) & \longrightarrow(X, A) \\
\left(e^{i \theta}, t\right) & \longmapsto\left(e^{i \theta}, t\right)
\end{aligned}
$$

Os levantamentos de $f$ têm a forma $\tilde{f}_{k}(\theta, t)=(\theta+2 k \pi, t), k \in \mathbb{Z}$, e as transformações de cobertura $\delta_{\ell}(\theta, t)=(\theta+2 \ell \pi, t), \ell \in \mathbb{Z}$. 
Portanto, temos que $R(f)=\infty$ (ou seja, $\#\left[\tilde{f}_{k}\right]=\infty$ ).

Similarmente, para o subespaço $A \subset X$, temos que $\left.f\right|_{A}=i d_{A}$ e os levantamentos de $\left.f\right|_{A}$ têm a forma $\tilde{f}_{A, r}(\theta)=\theta+2 \pi r, r \in \mathbb{Z}$.

Então, temos que $R\left(f_{A}\right)=\infty$.

Como

$$
L(f)=\sum_{q=0}^{1}(-1)^{q} \operatorname{tr} f_{*}=0 ; \quad \text { onde } \quad f_{*}: H_{q}(X, \mathbb{Q}) \rightarrow H_{q}(X, \mathbb{Q})
$$

e $X=S^{1} \times[0,1]$ é de Jiang, segue que $N(f)=0$, o que implica que todas as classes de levantamento de $f$ são inessenciais.

Similarmente, $L\left(f_{A}\right)=0$ e como $S^{1}$ é de Jiang, segue $N\left(f_{A}\right)=0$, e portanto, todas as classes de levantamento de $f_{A}$ inessenciais.

Observe que o diagrama

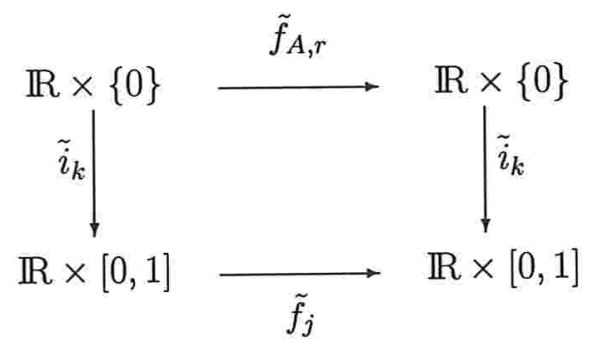

comuta se, e somente se,

$$
r=j \text { e } \tilde{i}_{k}(\theta, 0)=(\theta+2 k \pi, 0), k \in \mathbb{Z}
$$


Pela Proposição 2.1 ([15]), segue

$$
\left[\tilde{f}_{A, r}\right] \subset\left[\tilde{f}_{r}\right]
$$

ou seja, toda classe inessencial $\left[\tilde{f}_{r}\right]$ contém uma classe inessencial do tipo $\left[\tilde{f}_{A, r}\right]$.

Exemplo 1.2. Classe de levantamento inessencial de $f$ que contém classe de levantamento inessencial de $f_{A}$ :

Sejam $X=S^{1} \times[-1,1], A=S^{1} \times\{0\}$ e seja

$$
\begin{aligned}
f:(X, A) & \longrightarrow(X, A) \\
\left(e^{i \theta}, t\right) & \longmapsto\left(e^{i \theta},-t\right)
\end{aligned}
$$

As classes de levantamento de $f$ têm a forma $\tilde{f}_{k}(\theta, t)=(\theta+2 k \pi,-t), k \in \mathbb{Z}$, e as transformações de cobertura são dadas por $\delta_{\ell}(\theta, t)=(\theta+2 \ell \pi, t), \ell \in \mathbb{Z}$.

Temos então que $R(f)=\infty$ e $R\left(f_{A}\right)=\infty$, onde $\tilde{f}_{A, r}(\theta)=\theta+2 r \pi, r \in \mathbb{Z}$.

Como $L(f)=0$ e $X$ é de Jiang, temos que $N(f)=0$, ou seja, todas as classes $\left[\tilde{f}_{k}\right]$ são inessenciais.

Analogamente, para $f_{A}$, temos $L\left(f_{A}\right)=0$ e $S^{1}$ é de Jiang, logo $N\left(f_{A}\right)=0$, ou seja, todas as classes $\left[\tilde{f}_{A, r}\right]$ são inessenciais.

Também, neste caso, para cada $r \in \mathbb{Z}$, temos $\left[\tilde{f}_{A, r}\right] \subset\left[\tilde{f}_{r}\right]$, ou seja, temos $i_{r, F P C}:\left[\tilde{f}_{A, r}\right] \rightarrow\left[\tilde{f}_{r}\right]$ bijetora. 
Exemplo 1.3. Classe de levantamento essencial de $f$ que contém classe de levantamento essencial de $f_{A}$ e classe de levantamento essencial de $f$ que não contém classe de levantamento de $f_{A}$ :

Sejam $X=S^{1} \times[-1,1], A=\{(1,0)\}$ e a aplicação do par

$$
\begin{aligned}
f:(X, A) & \longrightarrow(X, A) \\
\left(e^{i \theta}, t\right) & \longmapsto\left(e^{-i \theta},-t\right)
\end{aligned}
$$

Os levantamentos de $f$ têm a forma $\tilde{f}_{s}(\theta, t)=(-\theta+2 s \pi,-t), s \in \mathbb{Z}$, e as transformações de cobertura são $\delta_{\ell}(\theta, t)=(\theta+2 \ell \pi, t), \ell \in \mathbb{Z}$.

Temos então, duas classes de levantamento $\left[\tilde{f}_{s}\right], s=0,1$, e assim, $R(f)=2$.

Como $f_{A}=i d_{A}=\tilde{f}_{A}$, segue que $R\left(f_{A}\right)=1$.

Os levantamentos $\tilde{i}_{k}$ têm a forma $\tilde{i}_{k}(1,0)=(2 k \pi, 0), k \in \mathbb{Z}$.

Consideremos então o diagrama

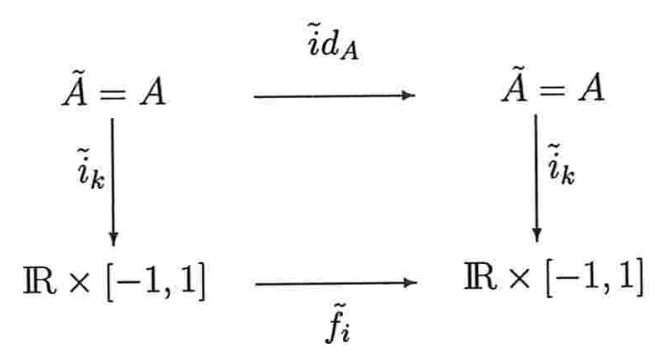

Para $i=0$, vale que $\left[\tilde{i d}_{A}\right] \subset\left[\tilde{f}_{0}\right]$, ou seja, $\tilde{i}_{F P C}\left(\left[\tilde{i d}_{A}\right]\right)=\left[\tilde{f}_{0}\right]$.

Para $i=1$, temos $\left[\widetilde{i d}_{A}\right] \not \subset\left[\tilde{f}_{1}\right]$.

Como $L(f)=2$ implica $N(f)=R(f)=2$, segue que as classes de levanta- 
mento $\left[\tilde{f}_{0}\right]$ e $\left[\tilde{f}_{1}\right]$ são essenciais.

Para $f_{A}$, temos também que $L\left(f_{A}\right)=1$, que implica $N\left(f_{A}\right)=R\left(f_{A}\right)=1$, pois $A$ é de Jiang. Portanto, $\left[\widetilde{i d}_{A}\right]$ é essencial.

Exemplo 1.4. Classe de levantamento essencial de $f$ que contém classe de levantamento essencial de $f_{A}$ e classe de levantamento essencial de $f$ que não contém classe de levantamento de $f_{A}$ :

Sejam $X=S^{1} \times[-1,1], \quad A=\{(-1,0)\}$ e

$$
\begin{aligned}
f:(X, A) & \longrightarrow(X, A) \\
\left(e^{i \theta}, t\right) & \longmapsto\left(e^{-i \theta},-t\right)
\end{aligned}
$$

Os levantamentos de $f$ têm a forma $\tilde{f}_{s}(\theta, t)=(-\theta+2 s \pi,-t), s \in \mathbb{Z}$, e as transformações de cobertura são $\delta_{\ell}(\theta, t)=(\theta+2 \ell \pi, t), \ell \in \mathbb{Z}, \#\left[\tilde{f}_{s}\right]=2$, $\#\left[\tilde{f}_{A}\right]=1$.

Como $L\left(f_{A}\right)=1$ e $A$ é de Jiang, $N\left(f_{A}\right)=R\left(f_{A}\right)$ e portanto $\left[\tilde{f}_{A}\right]$ é essencial.

Consideremos então o diagrama

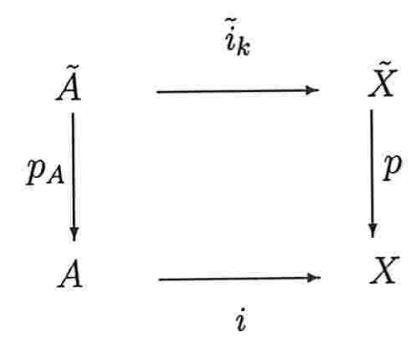


Temos que $\tilde{i}_{k}(-1,0)=(\pi+2 k \pi, 0), k \in \mathbb{Z}$. Por outro lado, o diagrama comuta para $k=0$ e $s=1$.

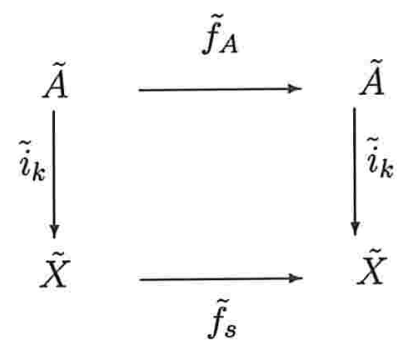

Para $k=0$, vale que $\left[\tilde{f}_{A}\right] \subset\left[\tilde{f}_{1}\right]$ e a outra classe $\left[\tilde{f}_{0}\right]$ não contém nada.

Como no exemplo anterior, as classes $\left[\tilde{f}_{0}\right]$ e $\left[\tilde{f}_{1}\right]$ são essenciais.

Exemplo 1.5. Classe de levantamento essencial de $f$ que contém classe de levantamento essencial de $f_{A}$ :

Sejam $X=S^{1} \times[-1,1], A=\{(1,0),(-1,0)\}$ e

$$
\begin{aligned}
f:(X, A) & \longrightarrow(X, A) \\
\left(e^{i \theta}, t\right) & \longmapsto\left(e^{-i \theta},-t\right)
\end{aligned}
$$

Os levantamentos de $f$ têm a forma $\tilde{f}_{s}(\theta, t)=(-\theta+2 s \pi,-t), s \in \mathbb{Z}$, e as transformações de cobertura são $\delta_{\ell}(\theta, t)=(\theta+2 \ell \pi, t), \ell \in \mathbb{Z}$.

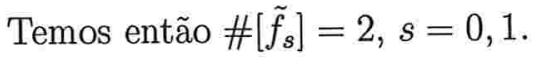

Para o subespaço $A=\{(1,0),(-1,0)\}=A_{1} \cup A_{2}$, temos

$$
\tilde{A}=A, \quad \tilde{f}_{A_{1}}=i d_{A_{1}} \text { e } \tilde{f}_{A_{2}}=i d_{A_{2}}
$$


ou seja, $\#\left[\tilde{f}_{A}\right]=2$, e são classes de levantamento essenciais.

Temos também que $\left[\tilde{f}_{0}\right]$ e $\left[\tilde{f}_{1}\right]$ são essenciais e vale que

$$
\left[\tilde{f}_{A_{2}}\right] \subset\left[\tilde{f}_{1}\right] \text { e }\left[\tilde{f}_{A_{1}}\right] \subset\left[\tilde{f}_{0}\right]
$$

Exemplo 1.6. Classe de levantamento essencial de $f$ que contém classe de levantamento inessencial de $f_{A}$ :

Consideremos $S^{2} \subset \mathbb{R}^{3}$ e os subespaços

$$
\begin{aligned}
& A_{1}=\{(\phi, \psi) ; 0 \leq \phi<2 \pi \text { e } 0 \leq \psi \leq \pi\} \\
& A_{2}=\{(\phi, \psi) ; 0 \leq \phi<2 \pi \text { e }-\pi \leq \psi \leq 0\} \\
& A_{0}=S^{1}=A_{1} \cap A_{2}
\end{aligned}
$$

Seja $f:\left(X ; A_{1}, A_{2}\right) \rightarrow\left(X, A_{1}, A_{2}\right)$ definida por $f(x)=x$ e sejam $f_{j}=\left.f\right|_{A_{j}}$, $j=1,2$.

Temos então que $f_{1}$ e $f_{2}$ possuem apenas uma classe de ponto fixo: $\mathbb{F}_{1}=A_{1}$ e $\mathbb{F}_{2}=A_{2}$ e $\mathbb{F}_{1}$ e $\mathbb{F}_{2}$ são essenciais, pois $L\left(f_{i}\right) \neq 0, i=1,2$, e como $A_{i}=i=1,2$ é de Jiang, segue $N\left(f_{i}\right)=R\left(f_{i}\right), i=1,2$.

Por outro lado, temos que $R\left(f_{0}\right)=\infty$ e $N\left(f_{0}\right)=0$, pois $L\left(f_{0}\right)=0$.

Pelo [12, Corolary 2.5] temos que, $\left(\mathbb{F}_{1}, \mathbb{F}_{2}\right)$ é um par inessencialmente unido 
de classes de pontos fixos essenciais. Portanto, essas duas classes essenciais só contêm classes inessenciais.

Exemplo 1.7. Classe de levantamento essencial de $f$ que contém classe de levantamento essencial de $f_{A}$ e classe de levantamento essencial de $f$ que não contém classe de levantamento de $f_{A}$ :

Sejam $X=S^{1}$ e $A=\{-1\} \subset S^{-1}$ e $f:(X, A) \rightarrow(X, A)$ definida por $f\left(e^{i t}\right)=e^{-i t}$ e $f_{A}=i d_{A}$.

Os levantamentos de $f$ têm a forma $\tilde{f}_{\ell}(t)=-t+2 \ell \pi ; \ell \in \mathbb{Z}$, e as transformações de cobertura são $\delta_{k}(t)=t+2 k \pi, k \in \mathbb{Z}$.

$$
\begin{aligned}
& \text { Temos que } \#\left[\tilde{f}_{\ell}\right]=2, \ell=0,1, \#\left[\tilde{f}_{A}\right]=1, \tilde{f}_{A}=i d_{A} \mathrm{e} \\
& {\left[\tilde{f}_{A}\right] \not \subset\left[\tilde{f}_{0}\right] \quad \text { e } \quad\left[\tilde{f}_{A}\right] \subset\left[\tilde{f}_{1}\right] .}
\end{aligned}
$$

Como $L(f)=2$ e $X$ é de Jiang, temos que $N(f)=R(f)$, ou seja, $\left[\tilde{f}_{0}\right]$ e $\left[\tilde{f}_{1}\right]$ são essenciais e $L\left(f_{A}\right)=1, \operatorname{logo} R\left(f_{A}\right)=N\left(f_{A}\right)=1$ e portanto $\left[\tilde{f}_{A}\right]$ é essencial.

Exemplo 1.8. Classe de levantamento inessencial de $f$ que contém classe de levantamento essencial de $f_{A}$ e classe de levantamento inessencial de $f$ que não contém classe de levantamento de $f_{A}$ : 
Seja $f:(X, A) \rightarrow(X, A)$ definida por $f(x)=x$, onde $X=S^{\prime}$ e $A=\{1\}$.

Como $L(f)=0$ e $S^{1}$ é espaço de Jiang, temos que $N(f)=0$, ou seja, todas as classes de levantamento de $f$ são inessenciais.

Para o subespaço $A=\{1\}$, temos

$$
\left[\tilde{f}_{A}\right]=i d ; \quad \tilde{f}_{A}(a)=1
$$

Portanto, $R\left(f_{A}\right)=N\left(f_{A}\right)=1$, pois $L\left(f_{A}\right)=1$ e assim $\left[\tilde{f}_{A}\right]$ é essencial. 


\section{Capítulo 2}

\section{Teorias de Reidemeister do Complemento e Relativa}

O objetivo inicial deste trabalho era definir novos números de Reidemeister para os contextos ainda não contemplados na literatura (veja o Capítulo 3). Entretanto, após os estudos iniciais verificou-se que seria possível melhorar os resultados obtidos em [2], para os números de Reidemeister Relativo e do Complemento. Tendo como base o tratamento adotado em [3] para a caracterização algébrica do Número de Reidemeister Relativo, modificamos a partição do conjunto das classes de levantamento apresentada em [2], como veremos a seguir.

Sejam $X$ um poliedro compacto e conexo, $A \subset X$ um subpoliedro finito (não necessariamente conexo) e $f:(X, A) \rightarrow(X, A)$ aplicação do par.

Se $A=\bigcup A_{i}$, onde $A_{i}$ são as componentes conexas de $A$, definimos $f_{A_{i}}=\left.f\right|_{A_{i}}$ e $\left.f\right|_{A}=f_{A}: A \rightarrow A$. 
Seja $C_{f}$ o conjunto das classes de levantamento de $f$ e consideremos a seguinte partição

$$
C_{f}=E_{f} \cup I_{f}
$$

onde:

$\diamond E_{f}$ é o conjunto das classes de levantamento essenciais, isto é, as $[\tilde{f}]$ tais que $p F i x(\tilde{f})$ é essencial.

$\diamond I_{f}$ é o conjunto das classes de levantamento inessenciais de $f$, isto é, as $[\tilde{f}]$ tais que $p F i x(\tilde{f})$ é inessencial ou vazia.

Da mesma maneira definimos $C_{f_{A_{i}}}=E_{f_{A_{i}}} \cup I_{f_{A_{i}}}$, para cada componente $A_{i}$ de $A$ e definimos:

$$
C_{f_{A}}=\bigcup_{i} C_{f_{A_{i}}}, \quad E_{f_{A}}=\bigcup_{i} E_{f_{A_{i}}}, \quad I_{f_{A}}=\bigcup_{i} I_{f_{A_{i}}}
$$

onde a reunião é feita para todo $i$ tal que $f\left(A_{i}\right) \subseteq A_{i}$.

Em relação à partição adotada, $C_{f}=E_{f} \cup I_{f}$, temos que o conjunto $E_{f}$ das classes de levantamento essenciais pode ser escrito como reunião disjunta de três conjuntos distintos:

$$
E_{f}=\left\{E_{f} \supset E_{f_{A}}\right\} \dot{\cup}\left\{E_{f} \supset I_{f_{A}}\right\} \dot{\cup}\left\{E_{f} \not \supset C_{f_{A}}\right\}
$$


onde:

$\diamond\left\{E_{f} \supset E_{f_{A}}\right\}$ denota o conjunto das classes de levantamento essenciais de $f$ que contém alguma classe essencial de $f_{A}$;

$\diamond\left\{E_{f} \supset I_{f_{A}}\right\}$ denota o conjunto das classes de levantamento essenciais de $f$ que só contém classes inessenciais de $f_{A}$;

$\diamond\left\{E_{f} \not \supset C_{f_{A}}\right\}$ denota o conjunto das classes de levantamento essenciais de $f$ que não contém nenhuma classe de $f_{A}$.

Analogamente, para o conjunto $I_{f}$, temos:

$$
I_{f}=\left\{I_{f} \supset E_{f_{A}}\right\} \dot{\cup}\left\{I_{f} \supset I_{f_{A}}\right\} \dot{\cup}\left\{I_{f} \not \supset C_{f_{A}}\right\}
$$

com as mesmas considerações anteriores.

Com essa partição do conjunto de classes de levantamento podemos então definir:

Definição 2.1. O conjunto de Reidemeister é definido por $r(f)=E_{f} \cup I_{f} e$ $o$ número de Reidemeister é $R(f)=\# E_{f}+\# I_{f}$. 
Definição 2.2. $O$ conjunto das classes de levantamento essenciais fracamente comum de $f$ e $f_{A}$ é definido por

$$
e\left(f, f_{A}\right)=\left\{E_{f} \supset C_{f_{A}}\right\}=\left\{E_{f} \supset E_{f_{A}}\right\} \cup\left\{E_{f} \supset I_{f_{A}}\right\}
$$

e sua cardinalidade é denotada por $E\left(f, f_{A}\right)=\#\left\{E_{f} \supset C_{f_{A}}\right\}$.

O número de Nielsen do complemento definido por Zhao em [15] pode ser descrito como:

Definição 2.3. Seja $f:(X, A) \rightarrow(X, A)$ aplicação do par. $O$ número de Nielsen do complemento $X-A$ é definido por

$$
N(f ; X-A)=N(f)-E\left(f, f_{A}\right)
$$

ou

$$
N(f ; X-A)=\# E_{f}-\#\left\{E_{f} \supset C_{f_{A}}\right\}
$$

O número de Nielsen do complemento tem as seguintes propriedades:

Teorema 2.1. Seja $(X, A)$ um par de poliedros compactos com $X$ conexo e seja $f:(X, A) \rightarrow(X, A)$ aplicação do par. Temos então: 
(1) $N(f ; X-A) \geq 0$;

(2) $N(f ; X-A)$ é um invariante homotópico;

(3) $N(f ; X-A) \leq \min \{\#$ Fix $g /$ Fix $g \cap(X-A) \neq \emptyset$ e $g \simeq f \operatorname{rel}(X, A)\}$;

(4) Se $f$ e g têm o mesmo tipo de homotopia, então $N(f ; X-A)=N(g ; Y-B)$;

(5) $N(f \circ g ; Y-B)=N(g \circ f ; X-A)$, onde

$$
f:(X, A) \rightarrow(X, A) \quad \text { e } g:(Y, B) \rightarrow(Y, B)
$$

e $f \circ g$ e $g \circ f$ estão bem definidas.

Demonstração: [15].

Vamos definir os conjuntos e números de Reidemeister do complemento e provar que esta definição reproduz, neste contexto, as propriedades usuais do número de Reidemeister:

Definição 2.4. Seja $f:(X, A) \rightarrow(X, A)$ aplicação do par. Os elementos de $\left\{C_{f} \supset C_{f_{A}}\right\}$ são chamados de classes de levantamento fracamente comum de $f$ e $f_{A}$ e denotamos este conjunto por $r\left(f, f_{A}\right)$, sendo sua cardinalidade denotada por $R\left(f, f_{A}\right)=\#\left\{C_{f} \supset C_{f_{A}}\right\}$. 
Definição 2.5. Seja $f:(X, A) \rightarrow(X, A)$ aplicação do par, onde $(X, A)$ é um par de poliedros compactos com $X$ conexo. Denotamos o conjunto de Reidemeister do complemento por $r(f ; X-A)$ e o definimos por

$$
r(f, X-A)=C_{f}-\left\{C_{f} \supset C_{f_{A}}\right\}
$$

Denotamos o número de Reidemeister do complemento por $R(f ; X-A) e$ o definimos como o número de classes de levantamento em $r(f ; X-A)$.

Assim,

$$
R(f ; X-A)=R(f)-R\left(f, f_{A}\right)
$$

ou

$$
R(f ; X-A)=\# C_{f}-\#\left\{C_{f} \supset C_{f_{A}}\right\}
$$

Se $R(f)$ ou $R\left(f, f_{A}\right)$ for infinito, dizemos que $R(f ; X-A)$ é infinito.

Na Teoria do ponto fixo, freqüentemente trabalhamos com espaços do tipo

$$
\hat{A}=\bigcup_{k}^{\circ} A_{k}
$$

onde $A_{k}$ são as componentes conexas do subespaço $A$ tais que $f\left(A_{k}\right) \subseteq A_{k}$.

Fazemos então a seguinte observação:

$$
\hat{A}=\emptyset \Longleftrightarrow R\left(f_{A}\right)=0 .
$$


De fato: Se $\hat{A}=\emptyset$, então, da definição de classe de levantamento, precisamos ter levantamento de auto-aplicações. Como não existe auto-aplicação, não existe levantamento e portanto $R\left(f_{A}\right)=0$.

Por outro lado, se existir uma componente de $A$ que admite $f_{A}$ como autoaplicação, temos que existe levantamento de $f_{A}$ nessa componente, logo temos pelo menos uma classe de levantamento, ou seja, $R\left(f_{A}\right) \neq 0$.

Observação 2.1. O número de Reidemeister do Complemento é uma generalizaçâo do número de Reidemeister $R(f)$, pois:

Seja $f:(X, A) \rightarrow(X, A)$ aplicação do par com $(X, A)$ par de poliedros compactos e $X$ conexo. Então

$$
R(f ; X-A)=R(f), \quad \text { se } \hat{A}=\emptyset \quad \text { ou } \quad A=\emptyset .
$$

De fato: $\hat{A}=\emptyset$ ou $A=\emptyset \Longleftrightarrow R\left(f_{A}\right)=0 \Longleftrightarrow C_{f_{A}}=\emptyset \Longleftrightarrow\left\{C_{f} \supset C_{f_{A}}\right\}=$ $\emptyset \Longleftrightarrow R\left(f, f_{A}\right)=0 \Longleftrightarrow R(f ; X-A)=R(f)$.

Proposição 2.1 (Limitante Superior). Seja $f:(X, A) \rightarrow(X, A)$ aplicação do par, onde $(X, A)$ é um par de poliedros compactos com $X$ conexo. Então

$$
R(R ; X-A) \geq N(f ; X-A)
$$


Demonstração: Temos

$$
\begin{aligned}
R(f ; X-A) & =\# r(f)-\# r\left(f, f_{A}\right) \\
& =\# E_{f}+\# I_{f}-\#\left\{E_{f} \supset C_{f_{A}}\right\}-\#\left\{I_{f} \supset C_{f_{A}}\right\} \\
& =\# E_{f}-\#\left\{E_{f} \supset C_{f_{A}}\right\}+\# I_{f}-\#\left\{I_{f} \supset C_{f_{A}}\right\} .
\end{aligned}
$$

Como $\left\{I_{f} \supset C_{f_{A}}\right\} \subset I_{f}$ segue $\# I_{f}-\#\left\{I_{f} \supset C_{f_{A}}\right\} \geq 0$.

Assim, $R(f ; X-A) \geq N(f ; X-A)$.

O número de Reidemeister do complemento satisfaz propriedades análogas às do número de Reidemeister clássico, como veremos a seguir:

Proposição 2.2 (Propriedade de Espaço de Jiang). Seja $(X, A)$ um par de poliedros compactos, onde $X$ é um espaço de Jiang conexo. Seja a aplicação do par $f:(X, A) \rightarrow(X, A), \operatorname{com} L(f) \neq 0$. Entẫo $R(f ; X-A)=N(f ; X-A)$.

Demonstração: O espaço $X$ é de Jiang e $L(f) \neq 0$, logo $N(f)=R(f)$. Daí,

$$
r\left(f, f_{A}\right)=\left\{C_{f} \supset C_{f_{A}}\right\}=\left\{E_{f} \supset C_{f_{A}}\right\}=e\left(f, f_{A}\right) .
$$


Portanto,

$$
R(f ; X-A)=R(f)-R\left(f, f_{A}\right)=N(f)-E\left(f, f_{A}\right)=N(f ; X-A) .
$$

Proposição 2.3 (Propriedade do Produto). Sejam $(X, A)$ e $(Y, B)$ pares de espaços, onde $X, Y, A, B$ são poliedros compactos e $X$ e $Y$ são espaços conexos.

Sejam $f:(X, A) \rightarrow(X, A)$ e $g:(Y, B) \rightarrow(Y, B)$ aplicações dos pares. Então

$$
R(f \times g ;(X \times Y)-(A \times B))=R(f) \cdot R(g)-R\left(f, f_{A}\right) \cdot R\left(g, g_{B}\right) .
$$

Demonstração: Por definição

$$
R(f \times g ;(X \times Y)-(A \times B))=R(f \times g)-R\left(f \times g, f_{A} \times g_{B}\right) .
$$

Como $R(f \times g)=R(f) \cdot R(g)$, basta mostrar que

$$
R\left(f, f_{A}\right) \cdot R\left(g, g_{B}\right)=R\left(f \times g, f_{A} \times g_{B}\right),
$$

onde

$$
\left\{\begin{array}{l}
R\left(f \times g, f_{A} \times g_{B}\right)=\#\left\{C_{(f \times g)} \supset C_{\left(f_{A} \times g_{B}\right)}\right\} \\
R\left(f, f_{A}\right)=\#\left\{C_{f} \supset C_{f_{A}}\right\} \\
R\left(g, g_{B}\right)=\#\left\{C_{g} \supset C_{g_{B}}\right\}
\end{array}\right.
$$


Existe uma correspondência entre os conjuntos

$$
r\left(f, f_{A}\right) \times r\left(g, g_{B}\right) \stackrel{\varphi}{\longleftrightarrow} r\left(f \times g, f_{A} \times g_{B}\right),
$$

ou seja,

$$
\left\{C_{f} \supset C_{f_{A}}\right\} \times\left\{C_{g} \supset C_{g_{B}}\right\} \stackrel{\varphi}{\longleftrightarrow}\left\{C_{(f \times g)} \supset C_{\left(f_{A} \times g_{B}\right)}\right\} .
$$

Seja $[\tilde{f}] \in\left\{C_{f} \supset C_{f_{A}}\right\}$. Então existe $\left[\tilde{f}_{A_{k}}\right]$, tal que $i_{k, F P C}\left[\tilde{f}_{A_{k}}\right]=[\tilde{f}]$ e seja $[\tilde{g}] \in\left\{C_{g} \supset C_{g_{B}}\right\}$. Logo, existe $\left[\tilde{g}_{B_{\ell}}\right]$ tal que $i_{\ell, F P C}\left[\tilde{g}_{B_{\ell}}\right]=[\tilde{g}]$.

Considere agora o seguinte diagrama:

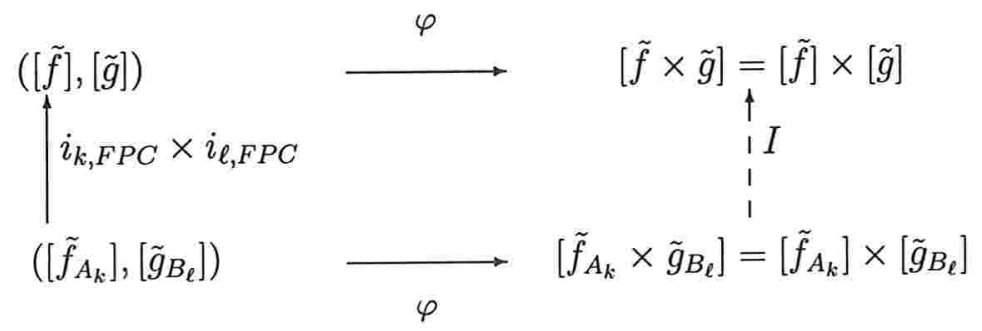

Definimos então

$$
I=i_{k l, F P C}=\left(i_{k, F P C}, i_{\ell, F P C}\right)
$$

Assim, o diagrama comuta e temos

$$
R\left(f, f_{A}\right) \cdot R\left(g, g_{B}\right)=R\left(f \times g, f_{A} \times g_{B}\right) .
$$


Portanto

$$
R(f \times g ;(X \times Y)-(A \times B))=R(f) \cdot R(g)-R\left(f, f_{A}\right) \cdot R\left(g, g_{B}\right) .
$$

Proposição 2.4 (Invariância Homotópica). Sejam $f, g:(X, A) \rightarrow(X, A)$ aplicações do par, onde $(X, A)$ é um par de poliedros compactos com $X$ conexo. Se f é homotópica a $g$ (rel. a A), então

$$
R(f ; X-A)=R(g ; X-A)
$$

Demonstração: Por definição, temos que:

$$
\left\{\begin{array}{l}
R(f ; X-A)=R(f)-R\left(f, f_{A}\right) \\
R(g ; X-A)=R(g)-R\left(g, g_{A}\right) .
\end{array}\right.
$$

Da teoria clássica de Nielsen, sabemos que se $f \simeq g$ então $R(f)=R(g)$.

Basta mostrar então que $R\left(f, f_{A}\right)=R\left(g, g_{A}\right)$, onde

$$
\left\{\begin{array}{l}
R\left(f, f_{A}\right)=\# r\left(f, f_{A}\right)=\#\left\{E_{f} \supset C_{f_{A}}\right\}+\#\left\{I_{f} \supset C_{f_{A}}\right\} \\
R\left(g, g_{A}\right)=\# r\left(g, g_{A}\right)=\#\left\{E_{g} \supset C_{g_{A}}\right\}+\#\left\{I_{g} \supset C_{g_{A}}\right\}
\end{array}\right.
$$

Em [15], Xuezhi Zhao mostrou que

$$
E\left(f, f_{A}\right)=\#\left\{E_{f} \supset C_{f_{A}}\right\}=\#\left\{E_{g} \supset C_{g_{A}}\right\}=E\left(g, g_{A}\right)
$$


Analogamente, definimos

$$
I\left(f, f_{A}\right)=\#\left\{I_{f} \supset C_{f_{A}}\right\} \text { e } I\left(g, g_{A}\right)=\#\left\{I_{g} \supset C_{g_{A}}\right\}
$$

Temos que

$$
I\left(f, f_{A}\right)=I\left(g, g_{A}\right)
$$

A demonstração é análoga a $\left(^{*}\right)$.

Em outras palavras, existe uma correspondência entre $I\left(f, f_{A}\right)$ e $I\left(g, g_{A}\right)$.

Portanto

$$
R(f ; X-A)=R(g ; X-A) .
$$

Helga Schirmer, em [11], descreveu de forma mais precisa o comportamento do número mínimo de pontos fixos de funções de pares de espaços, $M F[f ; X, A]$. Como o número de Nielsen usual, $N(f)$, não é um bom limitante inferior para $M F[f ; X, A]$, ela definiu o número de Nielsen relativo, $N(f ; X, A)$, como segue:

Definição 2.6. Seja $(X, A)$ um par de poliedros compactos, com $X$ conexo. Se $f:(X, A) \rightarrow(X, A)$ é uma aplicação do par, então o número de Nielsen 
relativo $N(f ; X, A)$ é definido como

$$
N(f ; X, A)=N(f)+N\left(f_{A}\right)-N\left(f, f_{A}\right)
$$

onde

$$
N\left(f, f_{A}\right)=\#\left\{E_{f} \supset E_{f_{A}}\right\} .
$$

Teorema 2.2 (Propriedades do número de Nielsen relativo).

(a) $0 \neq N(f ; X, A)<\infty$;

(b) $N(f ; X, A) \leq \# \operatorname{Fix}(f ; X, A)$;

(c) Se $(X, A)$ é um par de poliedros compactos e se são homotópicas as aplicações $f_{0}, f_{1}:(X, A) \rightarrow(X, A)$, então $N\left(f_{0} ; X, A\right)=N\left(f_{1} ; X, A\right)$;

(d) $N(f ; X, A) \leq \min \{\# \operatorname{Fix} g \mid g \simeq f \operatorname{rel} A\}$ (Nielsen relativo é um limitante inferior para o número de pontos fixos na classe de aplicaçôes homotópicas a $f \operatorname{rel} A)$;

(e) (Comutatividade): Sejam $(X, A)$ e $(Y, B)$ pares de poliedros compactos $e$ as aplicaçôes $f:(X, A) \rightarrow(Y, B)$ e $g:(Y, B) \rightarrow(X, A)$. Então tem-se $N(g \circ f ; X, A)=N(f \circ g ; Y, B)$ 
(f) (Invariante por tipo de homotopia): $\operatorname{Sejam}(X, A) e(Y, B)$ pares de poliedros compactos. Se as aplicações $f:(X, A) \rightarrow(X, A)$ e $g:(Y, B) \rightarrow(Y, B)$ têm o mesmo tipo de homotopia, então $N(f ; X, A)=N(g ; Y, B)$;

Demonstração: [11].

Definição 2.7. Seja $f:(X, A) \rightarrow(X, A)$ aplicação do par. Definimos

$$
R\left(f_{A}\right)=\# r\left(f_{A}\right)=\sum_{i} R\left(f_{A_{i}}\right)
$$

Observamos que:

$$
R\left(f_{A}\right) \geq N\left(f_{A}\right)=\sum_{i} N\left(f_{A_{i}}\right)
$$

Definição 2.8. O conjunto de Reidemeister Relativo, denotado por $r(f ; X, A)$

para $f:(X, A) \rightarrow(X, A)$ é definido como

$$
r(f ; X, A)=r\left(f_{A}\right) \cup r(f)-r\left(f, f_{A}\right)
$$

onde $r(f)=C_{f}, r\left(f_{A}\right)=C_{f_{A}}$ e $r\left(f, f_{A}\right)=\left\{C_{f} \supset C_{f_{A}}\right\}$.

Com respeito à partição adotada, $C_{f}=E_{f} \cup I_{f}$, temos

$$
r(f ; X, A)=C_{f} \cup C_{f_{A}}-\left\{C_{f} \supset C_{f_{A}}\right\} .
$$


Então, o número relativo de Reidemeister relativo é definido como

$$
R(f ; X, A)=R\left(f_{A}\right)+R(f)-R\left(f, f_{A}\right) .
$$

Se algum dos números $R(f), R\left(f_{A}\right)$ ou $R\left(f, f_{A}\right)$ é infinito, definimos então $R(f ; X, A)=\infty$. Caso contrário, temos $R(f ; X, A)<\infty$.

Com essa definição obtemos propriedades análogas às do número de Reidemeister, como veremos a seguir:

Proposição 2.5 (Limitante Superior). Seja $f:(X, A) \rightarrow(X, A)$ aplicação do par, onde X é poliedro compacto e conexo (finito). Então

$$
R(f ; X, A) \geq N(f ; X, A) .
$$

Demonstração: Com relação à partição $C_{f}=E_{f} \cup I_{f}$, temos que o conjunto de Nielsen relativo se escreve como $n(f ; X, A)=E_{f} \cup E_{f_{A}}-\left\{E_{f} \supset E_{f_{A}}\right\}$.

Por outro lado,

$$
\begin{aligned}
r(f ; X, A) & =C_{f} \cup C_{f_{A}}-\left\{C_{f} \supset C_{f_{A}}\right\} \\
& =E_{f} \cup I_{f} \cup E_{f_{A}} \cup I_{f_{A}}-\left(\left\{E_{f} \supset E_{f_{A}}\right\} \cup\left\{E_{f} \supset I_{f_{A}}\right\} \cup\left\{I_{f} \supset C_{f_{A}}\right\}\right)
\end{aligned}
$$

o que implica

$$
\# r(f ; X, A)=\# n(f ; X, A)+\# I_{f}-\#\left\{I_{f} \supset C_{f_{A}}\right\}+\# I_{f_{A}}-\#\left\{E_{f} \supset I_{f_{A}}\right\}
$$


Como $\# I_{f}-\#\left\{I_{f} \supset C_{f_{A}}\right\} \geq 0$ e $\# I_{f_{A}}-\#\left\{E_{f} \supset I_{f_{A}}\right\} \geq 0$ (visto que $\left.\#\left\{E_{f} \supset I_{f_{A}}\right\} \leq \# I_{f_{A}}\right)$ temos

$$
R(f ; X, A) \geq N(f ; X, A)
$$

Observação 2.2. Como $N\left(f, f_{A}\right)=\#\left\{E_{f} \supset E_{f_{A}}\right\}$, da definição de $R\left(f, f_{A}\right)$ segue $R\left(f, f_{A}\right) \geq N\left(f, f_{A}\right)$; o que torna impossivel demonstrar a Proposição 2.5 usando as partes de $R(f ; X, A)$.

Observação 2.3. O número de Reidemeister Relativo é uma generalização do número de Reidemeister clássico $R(f)$ :

Seja $f:(X, A) \rightarrow(X, A)$ aplicação do par, onde $X$ é um poliedro compacto $e$ conexo e $A \subset X$ é um subpoliedro. Então

$$
R(f ; X, A)=R(f)
$$

se $A=\emptyset$, ou $\hat{A}=\emptyset$, ou $A=X$.

De fato: Se $A=\emptyset$ ou $\hat{A}=\emptyset$ então $R\left(f_{A}\right)=0$ e $R\left(f, f_{A}\right)=0$, e segue que $R(f ; X, A)=R(f)$

Se $A=X$ então $R\left(f_{A}\right)=R(f)$ e $R\left(f, f_{A}\right)=\#\left\{C_{f} \supset C_{f_{A}}\right\}=\# C_{f}=R(f)$. 
Portanto, $R(f ; X, A)=R(f)+R(f)-R(f)=R(f)$.

Proposição 2.6. Seja $f:(X, A) \rightarrow(X, A)$ aplicação do par, com $(X, A)$ par de poliedros compactos, $X$ simplesmente conexo e $A \neq \emptyset$, ou $\hat{A} \neq \emptyset$. Então

$$
R(f ; X, A)=R\left(f_{A}\right)
$$

Demonstração: Como $X$ é simplesmente conexo, temos $R(f)=1=R\left(f, f_{A}\right)$.

Assim,

$$
R(f ; X, A)=R(f)+R\left(f_{A}\right)-R\left(f, f_{A}\right)=1+R\left(f_{A}\right)-1=R\left(f_{A}\right) .
$$

Proposição 2.7. Seja $f:(X, A) \rightarrow(X, A)$ aplicação do par, com $(X, A)$ par de poliedros compactos, $X$ conexo e A simplesmente conexo. Então

$$
R(f ; X, A)=R(f)
$$

Demonstração: Como $A$ é simplesmente conexo, $R\left(f_{A}\right)=1=R\left(f, f_{A}\right)$. Assim,

$$
R(f ; X, A)=R(f)+R\left(f_{A}\right)-R\left(f, f_{A}\right)=R(f)+1-1=R(f) .
$$


Proposição 2.8 (Propriedade de Espaço de Jiang). Seja $(X, A)$ um par de poliedros compactos, com $X$ conexo, onde $X$ e cada componente $A_{i}$ de $A$ são espaços de Jiang. Se $L(f) \neq 0$ e $L\left(f_{A_{i}}\right) \neq 0, \forall i$, tal que $f\left(A_{i}\right) \subset A_{i}$, então

$$
R(f ; X, A)=N(f ; X, A)
$$

Demonstração: Como $X$ é um espaço de Jiang, temos que $L(f) \neq 0$ implica $R(f)=N(f)$, isto é, $C_{f}=E_{f}$.

Similarmente, para as componentes $A_{i}^{\prime} \mathrm{s}: L\left(f_{A_{i}}\right) \neq 0$ e $A_{i}$ é espaço de Jiang, e temos portanto, $R\left(f_{A_{i}}\right)=N\left(f_{A_{i}}\right)$, isto é, $C_{f_{A_{i}}}=E f_{A_{i}}$, o que implica $C_{f_{A}}=E f_{A}$.

$$
\text { Assim }\left\{C_{f} \supset C_{f_{A}}\right\}=\left\{E_{f} \supset E_{f_{A}}\right\} \text { e segue que }
$$

$$
R(f ; X, A)=N(f ; X, A)
$$

Proposição 2.9 (Propriedade do Produto). Sejam $(X, A)$ e $(Y, B)$ pares de espaços, onde $X, Y, A, B$ são poliedros compactos, $X$ e $Y$ conexos. Sejam as funções $f:(X, A) \rightarrow(X, A)$ e $g:(Y, B) \rightarrow(Y, B)$. Então

$$
R(f \times g ; X \times Y, A \times B)=R\left(f_{A}\right) \cdot R\left(g_{B}\right)+R(f) \cdot R(g)-R\left(f, f_{A}\right) \cdot R\left(g, g_{B}\right) .
$$


Demonstração: Segue da Propriedade 1.2 (Propriedade do Produto), juntamente com o fato $R\left(f_{A} \times g_{B}\right)=R\left(f_{A}\right) \cdot R\left(g_{B}\right)$.

Proposição 2.10 (Invariância Homotópica). Sejam $f, g:(X, A) \rightarrow(X, A)$ aplicações do par, onde $(X, A)$ é um par de poliedros compactos com $X$ conexo. Se f é homotópica a $g$ (rel. a A), então

$$
R(f ; X, A)=R(g ; X, A) .
$$

Demonstração: Da teoria clássica temos que $R(f)=R(g)$ e $R\left(f_{A}\right)=R\left(g_{A}\right)$. Pela Proposição 2.4, temos $R\left(f, f_{A}\right)=R\left(g, g_{A}\right)$. Assim,

$$
R(f ; X, A)=R(g ; X, A)
$$




\section{Capítulo 3}

\section{O Número de Reidemeister da Tríade}

Uma tríade $\left(X, A_{1}, A_{2}\right)$ consiste de um espaço $X$ que é a reunião de dois subespaços $A_{1}$ e $A_{2}$. Observe que uma tríade $\left(X, A_{1}, A_{2}\right)$ se reduz a um par $\left(X, A_{1}\right)$, se $A_{2} \subset A_{1}$ ou $\left(X, A_{2}\right)$, se $A_{1} \subset A_{2}$.

Assim, uma aplicação de tríade $f:\left(X, A_{1}, A_{2}\right) \rightarrow\left(X, A_{1}, A_{2}\right)$ generaliza as aplicações dos pares.

Helga Schirmer em [12] definiu um novo número de Nielsen, denominado o número de Nielsen da tríade. Além das propriedades usuais para o número de Nielsen, ela demonstrou que para espaços que podem ser descritos como tríades, este novo número é melhor que o número de Nielsen Relativo para ser usado como um limitante inferior do número de pontos fixos na classe de aplicações homotópicas a $f: X \rightarrow X$. Se os espaços em questão obedecem certas condições, 
não muito limitantes, ela mostrou que o número de Nielsen da tríade realiza o número mínimo de pontos fixos na classe das aplicações homotópicas a $f$.

Neste capítulo definimos o número de Reidemeister da tríade, fundamentado na definição do número de Nielsen da tríade. Veremos que, devido ao seu enfoque ligado aos levantamentos, tal número de Reidemeister é um limitante superior para o respectivo número de Nielsen, usando apenas os números de Reidemeister Relativo, do Complemento, o clássico $R(f)$ e argumentos combinatórios. Outras propriedades usuais do número de Reidemeister da tríade são demonstrados no final deste capítulo, bem como alguns exemplos.

Sejam $X=A_{1} \cup A_{2}, A_{0}=A_{1} \cap A_{2}$ e $f:\left(X, A_{1}, A_{2}\right) \rightarrow\left(X, A_{1}, A_{2}\right)$ aplicação da tríade. Então, ficam determinadas as aplicações $f_{j}: A_{j} \rightarrow A_{j}, j=0,1,2$, onde $f_{j}=\left.f\right|_{A_{j}}$.

Definição 3.1. Sejam $f:\left(X, A_{1}, A_{2}\right) \rightarrow\left(X, A_{1}, A_{2}\right)$ uma aplicação da tríade $e$ $p_{j} \operatorname{Fix}\left(\tilde{f}_{j}\right)$ uma classe de pontos fixos de $f_{j}: A_{j} \rightarrow A_{j}$, para $j=1,2$. O par de classes de pontos fixos $\left(p_{1} \operatorname{Fix}\left(\tilde{f}_{1}\right), p_{2} \operatorname{Fix}\left(\tilde{f}_{2}\right)\right)$ denomina-se um par inessencialmente unido (de classes de pontos fixos) de $f_{1}$ e $f_{2}$ se as seguintes condições forem satisfeitas: 
(i) Existem uma componente $A_{0, k}$ de $A_{0}$ e uma classe inessencial de pontos fixos $p_{0, k} \operatorname{Fix}\left(\tilde{f}_{0, k}\right)$ de $f_{0, k}: A_{0, k} \rightarrow A_{0, k}$ que está contida em $p_{1} \operatorname{Fix}\left(\tilde{f}_{1}\right)$ e $p_{2} \operatorname{Fix}\left(\tilde{f}_{2}\right)$ (isto é: $i_{k, j, F P C}\left[\tilde{f}_{0, k}\right]=\left[\tilde{f}_{j}\right]$, para $\left.j=1,2\right)$.

(ii) Nenhuma classe essencial de pontos fixos $p_{0, k} \operatorname{Fix}\left(\tilde{f}_{0, k}\right)$ de qualquer aplicação $f_{0, k}: A_{0, k} \rightarrow A_{0, k}$ está contida em $p_{1} \operatorname{Fix}\left(\tilde{f}_{1}\right)$ e $p_{2} \operatorname{Fix}\left(\tilde{f}_{2}\right)$.

O par $\left(p_{1} \operatorname{Fix}\left(\tilde{f}_{1}\right), p_{2} \operatorname{Fix}\left(\tilde{f}_{2}\right)\right)$ é chamado um par inessencialmente unido de classes essenciais de pontos fixos de $f_{1}$ e $f_{2}$ se for um par inessencialmente unido e ambas as classes $p_{1} \operatorname{Fix}\left(\tilde{f}_{1}\right)$ e $p_{2} \operatorname{Fix}\left(\tilde{f}_{2}\right)$ forem essenciais. Denotamos o número de tais pares de classes por $I J\left(f_{1}, f_{2}\right)$.

Como o par $I J\left(f_{1}, f_{2}\right)$ possui boas propriedades, como invariância homotópica, comutatividade, invariância por tipo homotópico, Helga Schirmer definiu o número de Nielsen da tríade assim:

$$
N\left(f ; A_{1} \cup A_{2}\right)=N\left(f_{1}, A_{1}, A_{0}\right)+N\left(f_{2}, A_{2}, A_{0}\right)-N\left(f_{0}\right)-I J\left(f_{1}, f_{2}\right) .
$$

O novo número de Nielsen também possui as mesmas propriedades clássicas, e toda aplicação $f:\left(X, A_{1}, A_{2}\right) \rightarrow\left(X, A_{1}, A_{2}\right)$ possui, no mínimo, $N\left(f ; A_{1} \cup A_{2}\right)$ pontos fixos. 
Neste capítulo, definimos o número de Reidemeister da tríade em função de outros números de Reidemeister descritos no capítulo 2.

Este número possui também muitas propriedades como veremos a seguir.

Definição 3.2. Seja a tríade $\left(X, A_{1}, A_{2}\right)$, onde $X=A_{1} \cup A_{2}$ é um poliedro compacto e conexo, $A_{i}, i=1,2$, são subpoliedros e $A_{0}=A_{1} \cap A_{2}$. O número de Reidemeister da tríade é definido por

$$
R\left(f ; A_{1} \cup A_{2}\right)=R\left(f_{1} ; A_{1}, A_{0}\right)+R\left(f_{2} ; A_{2}, A_{0}\right)-R\left(f_{0}\right)
$$

A definição acima pode ser reescrita assim:

$$
R\left(f ; A_{1} \cup A_{2}\right)=R\left(f_{1} ; A_{1}-A_{0}\right)+R\left(f_{2} ; A_{2}, A_{0}\right)
$$

ou

$$
R\left(f ; A_{1} \cup A_{2}\right)=R\left(f_{1} ; A_{1}, A_{0}\right)+R\left(f_{2} ; A_{2}-A_{0}\right)
$$

ou

$$
R\left(f ; A_{1} \cup A_{2}\right)=R\left(f_{1} ; A_{1}-A_{0}\right)+R\left(f_{2} ; A_{2}-A_{0}\right)+R\left(f_{0}\right) .
$$




\subsection{Propriedades do número de Reidemeister da tríade}

Seja $f:\left(X, A_{1}, A_{2}\right) \rightarrow\left(X, A_{1}, A_{2}\right)$ aplicação da tríade. O número de Reidemeister da tríade possui as seguintes propriedades:

Teorema 3.1 (Limitante Superior). O número de Reidemeister da tríade é um limitante superior para o número de Nielsen da tríade, isto é:

$$
R\left(f ; A_{1} \cup A_{2}\right) \geq N\left(f ; A_{1} \cup A_{2}\right)
$$

Demonstração: Em relação à partição adotada temos, para $i=1,2$ :

$$
\begin{aligned}
& N\left(f_{i} ; A_{i}, A_{0}\right)=\# E_{f_{i}}+\# E_{f_{0}}-\#\left\{E_{f_{i}} \supset E_{f_{o}}\right\} \\
& R\left(f_{i} ; A_{i}, A_{0}\right)=\# C_{f_{i}}+\# C_{f_{0}}-\#\left\{C_{f_{i}} \supset C_{f_{o}}\right\}
\end{aligned}
$$

Então

$$
\begin{aligned}
R\left(f ; A_{1} \cup A_{2}\right)-N\left(f ; A_{1} \cup A_{2}\right)= & \#\left\{I_{f_{1}} \not \supset C_{f_{o}}\right\}+\#\left\{I_{f_{2}} \not \supset C_{f_{o}}\right\}+\# I_{f_{0}} \\
& -\#\left\{E_{f_{1}} \supset I_{f_{o}}\right\}-\#\left\{E_{f_{2}} \supset I_{f_{o}}\right\}+I J .
\end{aligned}
$$

Vamos provar que $\varepsilon=\# I_{f_{0}}-\#\left\{E_{f_{1}} \supset I_{f_{o}}\right\}-\#\left\{E_{f_{2}} \supset I_{f_{o}}\right\}+I J \geq 0$.

Para tanto, basta vermos como os elementos de $I_{f_{o}}$ se comportam em relação aos outros conjuntos em questão, uma vez que individualmente cada um deles 
tem cardinalidade menor ou igual à de $I_{f_{0}}$.

Observamos que toda classe de $I_{f_{0}}$ é uma $\left[\tilde{f}_{0, k}\right]$, para algum $k$, é inessencial e é levada pelas aplicações

$i_{k, F P C}^{1}:\left[\tilde{f}_{0, k}\right] \longmapsto\left[\tilde{f}_{1}\right]$, para alguma classe de levantamento de $\tilde{f}_{1}$

$i_{k, F P C}^{2}:\left[\tilde{f}_{0, k}\right] \longmapsto\left[\tilde{f}_{2}\right]$, para alguma classe de levantamento de $\tilde{f}_{2}$

Analisando os casos possíveis, vemos que cada classe de $I_{f_{0}}$ só contribui com 0 ou 1 à soma $\varepsilon$ :

- Se $i_{k, F P C}^{1}\left(\left[\tilde{f}_{0, k}\right]\right) \in I_{f_{1}}$ e $i_{k, F P C}^{2}\left(\left[\tilde{f}_{0, k}\right]\right) \in I_{f_{2}}$, então $\left[\tilde{f}_{0, k}\right]$ só é contada em $I_{f_{0}}$ e não afeta os outros termos de $\varepsilon$. Contribuição: 1 .

- Se $i_{k, F P C}^{1}\left(\left[\tilde{f}_{0, k}\right]\right) \in I_{f_{1}}$ e $i_{k, F P C}^{2}\left(\left[\tilde{f}_{0, k}\right]\right) \in E_{f_{2}}$, então $i_{k, F P C}^{2}\left(\left[\tilde{f}_{0, k}\right]\right)$ poderá ser contada em $\left\{E_{f_{2}} \supset I_{f_{o}}\right\}$, se a classe $i_{k, F P C}^{2}\left(\left[\tilde{f}_{0, k}\right]\right) \in E_{f_{2}}$ não foi contada antes e se não estiver na imagem $i_{j, F P C}^{2}$ de alguma $\left[\tilde{f}_{0, j}\right] \in E_{f_{0}}$, para algum $j$ (neste último caso $i_{k, F P C}^{2}\left(\left[\tilde{f}_{0, k}\right]\right) \in E_{f_{2}}$ seria contada em $\left\{E_{f_{2}} \supset E_{f_{o}}\right\}$ ). Contribuição: 0 ou 1. 
- Se $i_{k, F P C}^{1}\left(\left[\tilde{f}_{0, k}\right]\right) \in E_{f_{1}}$ e $i_{k, F P C}^{2}\left(\left[\tilde{f}_{0, k}\right]\right) \in I_{f_{2}}$, a conclusão é análoga ao item anterior.

- Se $i_{k, F P C}^{1}\left(\left[\tilde{f}_{0, k}\right]\right) \in E_{f_{1}}$ e $i_{k, F P C}^{2}\left(\left[\tilde{f}_{0, k}\right]\right) \in E_{f_{2}}$, temos que $\left[\tilde{f}_{0, k}\right]$ será contada em $I_{f_{0}}$. Podemos subdividir este caso em diferentes subcasos:

- Se $i_{k, F P C}^{1}\left(\left[\tilde{f}_{0, k}\right]\right)$ for contada em $\left\{E_{f_{1}} \supset I_{f_{o}}\right\}$ e $i_{k, F P C}^{2}\left(\left[\tilde{f}_{0, k}\right]\right)$ for contada em $\left.\left\{E_{f_{2}} \supset I_{f_{o}}\right\}\right)$, segue da partição adotada que $i_{k, F P C}^{1}\left(\left[\tilde{f}_{0, k}\right]\right) \not \supset E_{f_{0}}$ e $i_{k, F P C}^{2}\left(\left[\tilde{f}_{0, k}\right]\right) \not \supset E_{f_{0}}$, o que implica que $\left(i_{k, F P C}^{1}\left(\left[\tilde{f}_{0, k}\right]\right), i_{k, F P C}^{2}\left(\left[\tilde{f}_{0, k}\right]\right)\right)$ é um par essencialmente unido e portanto é contado em $I J$. Assim, contamos uma classe em $I_{f_{0}}$, uma classe em $\left\{E_{f_{1}} \supset I_{f_{o}}\right\}$, uma classe em $\left\{E_{f_{2}} \supset I_{f_{o}}\right\}$ e mais um par $I J$, totalizando zero.

- Os outros casos são análogos aos anteriores, com contribuição 0 ou 1 à soma.

Portanto, temos que

$$
R\left(f ; A_{1} \cup A_{2}\right) \geq N\left(f ; A_{1} \cup A_{2}\right)
$$


Proposição 3.1 (Invariância Homotópica). Sejam as aplicações da tríade $f, g:\left(X, A_{1}, A_{2}\right) \rightarrow\left(X, A_{1}, A_{2}\right)$. Se $f$ é homotópica a $g$ por uma homotopia que restrita ao par $\left(A_{1}, A_{0}\right)$ é rel. $A_{0}$, e que restrita ao par $\left(A_{2}, A_{0}\right)$ é rel. $A_{0}$, então

$$
R\left(f ; A_{1} \cup A_{2}\right)=R\left(g ; A_{1} \cup A_{2}\right)
$$

Demonstração: Por definição, temos:

$$
\begin{aligned}
& R\left(f ; A_{1} \cup A_{2}\right)=R\left(f_{1} ; A_{1}, A_{0}\right)+R\left(f_{2} ; A_{2}, A_{0}\right)-R\left(f_{0}\right), \\
& R\left(g ; A_{1} \cup A_{2}\right)=R\left(g_{1} ; A_{1}, A_{0}\right)+R\left(g_{2} ; A_{2}, A_{0}\right)-R\left(g_{0}\right) .
\end{aligned}
$$

Como os números de Reidemeister e Reidemeister Relativo são invariantes homotópicos, temos $R\left(f_{j} ; A_{j}, A_{0}\right)=R\left(g_{j} ; A_{j}, A_{0}\right), j=1,2$, e

$$
R\left(f_{0}\right)=R\left(g_{0}\right) \Rightarrow R\left(f ; A_{1} \cup A_{2}\right)=R\left(g ; A_{1} \cup A_{2}\right) .
$$

Observação 3.1. O número de Reidemeister da tríade $R\left(f ; A_{1} \cup A_{2}\right)$ generaliza o número de Reidemeister clássico, $R(f)$, e o número de Reidemeister Relativo, como veremos a seguir:

(i) Se $A_{0}=\emptyset$ ou se $\hat{A}_{0}=\emptyset$, então $R\left(f ; A_{1} \cup A_{2}\right)=R\left(f_{1}\right)+R\left(f_{2}\right)$. 
De fato: Pela Observação 2.3, temos que $R\left(f_{i} ; A_{i}, A_{0}\right)=R\left(f_{i}\right), i=1,2$, e pelos comentários anteriores à Observação 2.1, $R\left(f_{0}\right)=0$. Assim,

$$
R\left(f ; A_{1} \cup A_{2}\right)=R\left(f_{1} ; A_{1}, A_{0}\right)+R\left(f_{2} ; A_{2}, A_{0}\right)-R\left(f_{0}\right)=R\left(f_{1}\right)+R\left(f_{2}\right) .
$$

(ii) Se $A_{1}=A_{2}$, então $R\left(f ; A_{1} \cup A_{2}\right)=R(f)$.

De fato: Como $A_{1}=A_{2}, f_{1}=f_{2}=f=f_{0}$ e $R\left(f_{1}\right)=R\left(f_{2}\right)=R\left(f_{0}\right)=$ $R(f)$, pela Observação 2.3 , temos $R\left(f_{i} ; A_{i}, A_{0}\right)=R\left(f_{i}\right)=R(f), i=1,2$.

Assim

$$
\begin{aligned}
R\left(f ; A_{1} \cup A_{2}\right) & =R\left(f_{1} ; A_{1}, A_{0}\right)+R\left(f_{2} ; A_{2}, A_{0}\right)-R\left(f_{0}\right) \\
& =R(f)+R(f)-R\left(f_{0}\right)=R(f) .
\end{aligned}
$$

(iii) Se $A_{2} \subset A_{1}$, então $R\left(f ; A_{1} \cup A_{2}\right)=R\left(f_{1} ; A_{1}, A_{0}\right)$.

De fato: Como $A_{2} \subset A_{1}$, segue $A_{0}=A_{1} \cap A_{2}=A_{2}$ e $f_{2}=\left.f\right|_{A_{2}}=f_{0}$.

Então:

$$
R\left(f ; A_{1} \cup A_{2}\right)=R\left(f_{1} ; A_{1}, A_{0}\right)+R\left(f_{0} ; A_{0}, A_{0}\right)-R\left(f_{0}\right) .
$$

$\operatorname{Mas} R\left(f_{0} ; A_{0}, A_{0}\right)=R\left(f_{0}\right)+R\left(f_{0}\right)-R\left(f_{0}, f_{0}\right)=R\left(f_{0}\right)$. Portanto

$$
R\left(f ; A_{1} \cup A_{2}\right)=R\left(f_{1} ; A_{1}, A_{0}\right) .
$$


Analogamente, se $A_{1} \subset A_{2}$, então

$$
R\left(f ; A_{1} \cup A_{2}\right)=R\left(f_{2} ; A_{2}, A_{0}\right) .
$$

Uma propriedade importante é obtida quando trabalhamos com espaços topológicos $X=A_{1} \cup A_{2}$, com $A_{j}$ simplesmente conexo, $j=1$, 2, e $A_{1} \cap A_{2}$ conexo por caminhos. O teorema de Van-Kampen nos diz então que $X$ é simplesmente conexo.

Então, se $f: X \rightarrow X$ ( $X$ nas condições anteriores), sabemos da teoria clássica de Nielsen que $R\left(f_{j}\right)=1, j=1,2$, e o cálculo do número de Reidemeister da tríade $R\left(f ; A_{1} \cup A_{2}\right)$ se reduz ao cálculo do número de classes de levantamento de $\left.f\right|_{A_{0}}=f_{0}$.

Proposição 3.2. Se $f:\left(X, A_{1}, A_{2}\right) \rightarrow\left(X, A_{1}, A_{2}\right)$ é aplicação da tríade com $A_{j}$ simplesmente conexo, $j=1,2$, e $A_{0}=A_{1} \cap A_{2}, A_{0} \neq \emptyset\left(\right.$ ou $\hat{A}_{0} \neq \emptyset$ ), então

$$
R\left(f ; A_{1} \cup A_{2}\right)=R\left(f_{0}\right)
$$

Demonstração: Pela Proposição 2.6, temos que: $R\left(f_{i} ; A_{i}, A_{0}\right)=R\left(f_{0}\right), i=1,2$. 
Portanto,

$$
R\left(f ; A_{1} \cup A_{2}\right)=R\left(f_{0}\right)+R\left(f_{0}\right)-R\left(f_{0}\right)=R\left(f_{0}\right) .
$$

Seja $f:\left(X, A_{1}, A_{2}\right) \rightarrow\left(X, A_{1}, A_{2}\right)$ aplicação da tríade, onde $A_{0}=A_{1} \cap A_{2}$. Temos então:

Proposição 3.3. Se $A_{0}$ é simplemente conexo, então

$$
R\left(f ; A_{1} \cup A_{2}\right)=R\left(f_{1}\right)+R\left(f_{2}\right)-1 .
$$

Demonstração: Como $A_{0}$ é simplesmente conexo, $R\left(f_{0}\right)=1$ e pela Proposição

2.7 temos que $R\left(f_{i} ; A_{i}, A_{0}\right)=R\left(f_{i}\right), i=1,2$. Assim,

$$
R\left(f ; A_{1} \cup A_{2}\right)=R\left(f_{1}\right)+R\left(f_{2}\right)-1 .
$$

Proposição 3.4. (i) Se $A_{1}$ é simplesmente conexo, então

$$
R\left(f ; A_{1} \cup A_{2}\right)=R\left(f_{2} ; A_{2}, A_{0}\right)
$$

(ii) $\mathrm{Se} \mathrm{A}_{2}$ é simplesmente conexo, então

$$
R\left(f ; A_{1} \cup A_{2}\right)=R\left(f_{1} ; A_{1}, A_{0}\right) .
$$




\section{Demonstração:}

(i) Se $A_{1}$ é simplesmente conexo, pela Proposição 2.6 segue $R\left(f_{1} ; A_{1}, A_{0}\right)=R\left(f_{0}\right)$. Assim,

$$
R\left(f ; A_{1} \cup A_{2}\right)=R\left(f_{0}\right)+R\left(f_{2} ; A_{2}, A_{0}\right)-R\left(f_{0}\right)=R\left(f_{2} ; A_{2}, A_{0}\right)
$$

(ii) Demonstração análoga.

Proposição 3.5 (Espaços de Jiang). Seja $f:\left(X, A_{1}, A_{2}\right) \rightarrow\left(X, A_{1}, A_{2}\right)$ aplicação da tríade. Se $A_{i}$ é espaço de Jiang e $L\left(f_{i}\right) \neq 0, i=0,1,2$, então

$$
R\left(f ; A_{1} \cup A_{2}\right)=N\left(f ; A_{1} \cup A_{2}\right) .
$$

Demonstração: Como $A_{i}$ é o espaço de Jiang e $L\left(f_{i}\right) \neq 0, i=0,1,2$, temos que $R\left(f_{j} ; A_{j}, A_{0}\right)=N\left(f_{j} ; A_{j}, A_{0}\right), j=1,2$, e $R\left(f_{0}\right)=N\left(f_{0}\right)$, o que implica que $I J\left(f_{1}, f_{2}\right)=0$. Logo, $R\left(f ; A_{1} \cup A_{2}\right)=N\left(f ; A_{1} \cup A_{2}\right)$.

\subsection{Exemplos}

O cálculo do número de Reidemeister da tríade (ou do número de Nielsen da tríade) se reduz, como vimos, ao cálculo de outros números de Reidemeister (ou de Nielsen) já descritos na Teoria do Ponto Fixo de Nielsen. 
A seguir, apresentamos alguns exemplos geométricos usando o número de Reidemeister da tríade.

Exemplo 3.1 (O Duplo de uma variedade). Seja $M$ uma variedade compacta, conexa, triangulável e com fronteira $\partial M$ e seja $D M=M_{+} \cup M_{-}$o seu duplo e $f:(M, \partial M) \rightarrow(M, \partial M)$ a aplicação do par. A aplicação

$$
D f:\left(D M, M_{+}, M_{-}\right) \rightarrow\left(D M, M_{+}, M_{-}\right)
$$

é uma aplicação da tríade.

Calculemos $R\left(D f ; M_{+} \cup M_{-}\right)$.

Seja $D f_{1}=\left.D f\right|_{M_{+}}$e $D f_{2}=\left.D f\right|_{M_{-}}$. Como $M_{+}$é homeomorfo a $M_{-}$, temos que $D f_{1}=D f_{2}$.

Assim,

$$
\begin{aligned}
R\left(D f ; M_{+} \cup M_{-}\right) & =2 R\left(D f_{1}\right)-2 R\left(D f_{1}, D f_{0}\right)+R\left(D f_{0}\right) \\
& =R\left(D f_{1} ; M_{+}, \partial M\right)+R\left(D f_{1} ; M_{+}-\partial M\right) .
\end{aligned}
$$

Exemplo 3.2 (A Suspensão de um Poliedro). Seja $X$ um poliedro compacto e sejam $X_{+}$e $X_{-}$cones sobre $X, S X=X_{+} \cup X_{-}$a suspensão de $X$. A aplicação 
$f: X \rightarrow X$ nos define a aplicação da tríade

$$
S f:\left(S X, X_{+} \cup X_{-}\right) \rightarrow\left(S X, X_{+} \cup X_{-}\right)
$$

Por definição

$$
R\left(S f ; X_{+} \cup X_{-}\right)=R\left(S f_{1}, X_{+}, X_{0}\right)+R\left(S f_{2}, X_{-}, X_{0}\right)-R\left(S f_{0}\right)
$$

onde $S f_{1}=\left.S f\right|_{X_{+}}, S f_{2}=\left.S f\right|_{X_{-}}$e $S f_{0}=\left.S f\right|_{X}=f$.

Como $X_{+}$e $X_{-}$são simplesmente conexos, temos pela Proposição 2.6 que

$$
R\left(S f_{1}, X_{+}, X_{0}\right)=R\left(S f_{0}\right) \text { e } R\left(S f_{2}, X_{-}, X_{0}\right)=R\left(S f_{0}\right)
$$

Assim,

$$
\begin{aligned}
R\left(S f ; X_{+} \cup X_{-}\right) & =R\left(S f_{1}, X_{+}, X_{0}\right)+R\left(S f_{2}, X_{-}, X_{0}\right)-R\left(S_{f_{0}}\right) \\
& =R\left(S f_{0}\right)+R\left(S f_{0}\right)-R\left(S f_{0}\right)=R\left(S f_{0}\right)=R(f) .
\end{aligned}
$$

Exemplo 3.3 (Colando p-alças). Seja $M=M_{1} \cup H$ a variedade obtida de $M_{1}$, onde $M_{1}$ é uma $n$-variedade compacta, conexa, triangulável $(n \geq 2)$, com fronteira, colando em sua fronteira uma p-alça $\mathrm{H}$, homeomorfa à imagem de $I^{p} \times$ $I^{q}, p+q=n$. Temos então: $\left(M, M_{1}, H\right)$ é uma tríade, com $M_{0}=M_{1} \cap H=$ 
$\left(\partial I^{p}\right) \times I^{q}$ e $f:\left(M, M_{1}, H\right) \rightarrow\left(M, M_{1}, H\right)$ é aplicação de tríade, com $f_{0}=\left.f\right|_{M_{0}}$.

Como $H \cong I^{p} \times I^{q}, \operatorname{com} p+q=n \geq 2$ que é 1 -conexo temos que $R\left(f_{2}\right)=1$.

Para obter a expressão de $R\left(f ; M_{1} \cup H\right)$ dividimos nos seguintes casos:

1. Se $p=1$ :

Temos $M_{0}=\{p, q\} \times I^{n-1} \cong B^{n-1} \dot{\cup} B^{n-1}=$ união disjunta de duas $(n-1)$ bolas.

(a) Se $\hat{M}_{0}=\emptyset$ então pela Observação 3.1(i) temos $R\left(f ; M_{1} \cup H\right)=R\left(f_{1}\right)+$ $R\left(f_{2}\right)=R\left(f_{1}\right)+1$.

(b) Se $\hat{M}_{0}=I^{n-1}$ então pela Proposição 3.3 temos $R\left(f ; M_{1} \cup H\right)=R\left(f_{1}\right)+$ $R\left(f_{2}\right)-1=R\left(f_{1}\right)$

(c) Se $\hat{M}_{0}=M_{0}$ então temos que $R\left(f_{0}\right)=2$ e da definição obtemos $R\left(f ; M_{1} \cup H\right)=R\left(f_{1} ; M_{1}-M_{0}\right)+R\left(f_{2} ; M_{2}, M_{0}\right)=R\left(f_{1} ; M_{1}-M_{0}\right)+2$.

2. Se $p=2$ :

(a) Se $n=2$ então $M_{0}=S^{1} \times\{p, q\} \cong S^{1} \cup \dot{U} S^{1}=$ união disjunta de dois $S^{1}$. 
i. Se $\hat{M}_{0}=\emptyset$ então pela Observação 3.1(i) temos $R\left(f ; M_{1} \cup H\right)=$

$$
R\left(f_{1}\right)+R\left(f_{2}\right)=R\left(f_{1}\right)+1
$$

ii. Se $\hat{M}_{0}=S^{1}$ ou $\hat{M}_{0}=M_{0}$ então obtemos da definição $R\left(f ; M_{1} \cup\right.$

$$
H)=R\left(f_{1} ; M_{1}-M_{0}\right)+R\left(f_{2} ; M_{2}, M_{0}\right)=R\left(f_{1} ; M_{1}-M_{0}\right)+R\left(f_{0}\right) .
$$

(b) Se $n \geq 3$ então $M_{0}=S^{1} \times I^{n-2}$ então obtemos da definição $R\left(f ; M_{1} \cup\right.$

$$
H)=R\left(f_{1} ; M_{1}-M_{0}\right)+R\left(f_{2} ; M_{2}, M_{0}\right)=R\left(f_{1} ; M_{1}-M_{0}\right)+R\left(f_{0}\right) .
$$

3. Se $p \geq 3$ temos que $\partial I^{p}$ é simplesmente conexo, $\log M_{0}$ é simplesmente conexo e pela Proposição 3.3 temos $R\left(f ; M_{1} \cup H\right)=R\left(f_{1}\right)+R\left(f_{2}\right)-1=$ $R\left(f_{1}\right)$.

Exemplo 3.4 (Somas Conexas). Consideremos o caso $M=M_{1} \# M_{2}$ a soma conexa de duas $n$-variedades compactas, conexas e trianguláveis, (para $n \geq 3$ ), isto é, $M$ é obtida removendo-se o interior $\operatorname{Int} B$ de uma $n$-bola $B$ de $M_{j}, j=1,2$, e identificando as fronteiras das variedades resultantes com o "buraco" $M_{j}^{0}=$ $M_{j}-\operatorname{Int} B$.

Portanto, $A_{0}=M_{1}^{0} \cap M_{2}^{0}$ é uma $(n-1)$-esfera.

Seja $f:\left(M, M_{1}^{0}, M_{2}^{0}\right) \rightarrow\left(M, M_{1}^{0}, M_{2}^{0}\right)$ a aplicação da tríade, onde $\left.f\right|_{M_{1}^{0}}=f_{1}$, 
$\left.f\right|_{M_{2}^{0}}=f_{2}$.

Observamos que neste caso, como $n \geq 3$ e $M_{0} \approx S^{n-1}$, segue que $M_{0}$ é simplesmente conexo. Daí, pela Proposição 3.3 temos

$$
R\left(f ; M_{1}^{0} \cup M_{2}^{0}\right)=R\left(f_{1}\right)+R\left(f_{2}\right)-1 .
$$

Exemplo 3.5. Sejam $D^{2}$ o disco unitário, $\partial D^{2}=S^{1}$. Consideremos

$$
f: \begin{aligned}
\left(D^{2}, S^{1}\right) & \longrightarrow\left(D^{2}, S^{1}\right) \\
\left(r e^{i \theta}, e^{i t}\right) & \longmapsto\left(r e^{3 i \theta}, e^{3 i t}\right)
\end{aligned}
$$

com $0 \leq r \leq 1$, e seja $D M$ o duplo de $D^{2}$. Temos que

$$
R\left(D f ; M_{+} \cup M_{-}\right)=2
$$

pois $R\left(D f_{0}\right)=2, R\left(D f_{j}\right)=1$ e $R\left(D f_{j}, D f_{0}\right)=1, j=1,2$.

Como apresentado em [12], $N\left(D f ; M_{+} \cup M_{-}\right)=1$, portanto, o número de Reidemeister da tríade pode ser uma boa aproximação para o número de Nielsen da tríade quando a dimensão do espaço é 2 .

Exemplo 3.6. Sejam $X=S^{2}$ a esfera unitária do $\mathbb{R}^{3}$ e

$$
A_{1}=\{(\phi, \psi): 0 \leq \phi<2 \pi, 0 \leq \psi \leq \pi\}
$$




$$
A_{2}=\{(\phi, \psi): 0 \leq \phi<2 \pi,-\pi \leq \psi \leq 0\}
$$

Se $f:\left(X, A_{1}, A_{2}\right) \rightarrow\left(X, A_{1}, A_{2}\right)$ é a suspensão de uma aplicação $f_{0}$ de grau $d \neq 1$, temos então que $R\left(f ; A_{1} \cup A_{2}\right)=R\left(f_{0}\right)=|1-d|$.

Em [12], Helga Schirmer calculou o número $N\left(f ; A_{1} \cup A_{2}\right)$ que é exatamente igual ao número de Reidemeister $R\left(f ; A_{1} \cup A_{2}\right)$. 


\section{Capítulo 4}

\section{Caracterização Algébrica do Número de Reidemeister da Tríade}

Neste capítulo descrevemos caracterizações algébricas do número de Reidemeister da tríade. Na primeira parte apresentamos o conceito de $\varphi$-classe de conjugação, com bibliografia básica [7] e aplicações à teoria Relativa de Nielsen, através da definição de uma caracterização algébrica para o número de Reidemeister Relativo (ver [3]; para aplicações em pares fibrados que preservam fibras, ver [4]). Utilizando as definições dadas em [3], obtemos uma primeira caracterização algébrica para $R\left(f ; A_{1} \cup A_{2}\right)$. Na segunda parte, seguindo os passos de Zhao, que em [15] obteve fórmulas algébricas para $N(f ; X, A)$ e $N(f ; X-A)$ no caso de $X$ e $A_{k} \subset \hat{A}$ serem espaços de Jiang, obtemos uma outra formulação algébrica para o número de Reidemeister da tríade. 


\subsection{Formulação Algébrica por $\varphi$-conjugação}

Sejam $X$ um poliedro compacto e conexo, $A \subset X$ um subpoliedro finito (não necessariamente compacto), $f:(X, A) \rightarrow(X, A)$ aplicação do par e $\eta_{X}: \tilde{X} \rightarrow X$ a aplicação de revestimento de $X$.

Seja $\tilde{f}$ um levantamento de $f$. Então, para cada $\sigma \in \operatorname{Cov}\left(\eta_{X}\right) \cong \pi_{1}(X)$, existe um único elemento $\varphi(\sigma) \in \pi_{1}(X)$ tal que

$$
\tilde{f} \sigma=\varphi(\sigma) \tilde{f}
$$

A correspondência

$$
\sigma \stackrel{\varphi}{\longmapsto} \varphi(\sigma)
$$

é um homomorfismo de grupos fundamentais.

Similarmente, para cada componente $A_{k}$ de $A$, tal que $f\left(A_{k}\right) \subseteq A_{k}$, temos que para cada $\sigma \in \operatorname{Cov}\left(\eta_{A_{k}}\right) \cong \pi_{1}\left(A_{k}\right)$ e $\tilde{f}_{k}$ levantamento de $f_{k}=\left.f\right|_{A_{k}}$, existe um único elemento $\varphi_{k}(\sigma) \in \pi_{1}\left(A_{k}\right)$ tal que

$$
\tilde{f}_{k} \sigma=\varphi_{k}(\sigma) \tilde{f}_{k}
$$

e a correspondência $\sigma \longmapsto \varphi_{k}(\sigma)$ é um homomorfismo de grupos fundamentais.

Como cada levantamento de $f$ é da forma $\alpha \tilde{f}\left(\alpha \in \operatorname{Cov}\left(\eta_{X}\right)\right.$ e $\tilde{f}$ fixado), 
temos:

$$
[\alpha \tilde{f}]=[\beta \tilde{f}] ; \quad \alpha, \beta \in \operatorname{Cov}\left(\eta_{X}\right) \Longleftrightarrow \beta \tilde{f}=\sigma \alpha \tilde{f} \sigma^{-1}=\sigma \alpha \varphi\left(\sigma^{-1}\right) \tilde{f}
$$

e portanto $\beta=\sigma \alpha \varphi\left(\sigma^{-1}\right)$.

Dizemos então que os elementos $\alpha, \beta \in \operatorname{Cov}\left(\eta_{X}\right) \cong \pi_{1}(X)$ são $\varphi$-conjugados e denotaremos por $[\sigma]$ a $\varphi$-classe de conjugação de $\sigma \in \pi_{1}(X)$.

Pelas observações anteriores podemos então enunciar o seguinte teorema:

Teorema 4.1. As classes de levantamento de $f$ estão em correspondência injetora com as $\varphi$-classes de conjugação de $\pi_{1}(X)$.

Demonstração: [7].

Consideremos a seguinte ação (à esquerda) em $\pi_{1}(X)$ :

$$
\begin{aligned}
\pi_{1}(X) \times \pi_{1}(X) & \longrightarrow \pi_{1}(X) \\
(\alpha, \sigma) & \longmapsto \alpha \cdot \sigma=\alpha \sigma \varphi\left(\alpha^{-1}\right)
\end{aligned}
$$

Temos então que: a órbita de $\sigma$ é o conjunto $\left\{\alpha \sigma \varphi\left(\alpha^{-1}\right) ; \alpha \in \pi_{1}(X)\right\}$.

Assim, $\beta$ pertence à órbita de $\sigma \Longleftrightarrow \beta=\alpha \sigma \varphi\left(\alpha^{-1}\right) \Longleftrightarrow[\beta \tilde{f}]=[\sigma \tilde{f}]$. 
Definição 4.1. Definimos o conjunto das classes de Reidemeister $\mathcal{R}(\varphi, \pi)$ por:

$$
\mathcal{R}\left(\varphi, \pi_{1}\right)=\frac{\pi_{1}(X)}{\sim}
$$

onde $\sim$ é a relação obtida por $\varphi$-conjugação (ou o conjunto das órbitas da ação por $\varphi$-conjugação).

Ao juntarmos a Definição 4.1 com as observações anteriores, podemos dizer que:

$$
R(f)=\# \mathcal{R}\left(\varphi, \pi_{1}\right)
$$

Similarmente, para cada componente $A_{k}$, tal que $f\left(A_{k}\right) \subseteq A_{k}$, temos o conjunto das classes de Reidemeister $\mathcal{R}\left(\varphi_{k}, \pi_{1}\left(A_{k}\right)\right)$ e $R\left(f_{k}\right)=\# \mathcal{R}\left(\varphi_{k}, \pi_{1}\left(A_{k}\right)\right)$.

Definimos então

$$
\mathcal{R}\left(\varphi_{A}, \pi_{1}(A)\right)=\bigcup_{k} \mathcal{R}\left(\varphi_{k}, \pi_{1}\left(A_{k}\right)\right)
$$

e

$$
R\left(f_{A}\right)=\# \mathcal{R}\left(\varphi_{A}, \pi_{1}(A)\right)=\sum_{k} \# \mathcal{R}\left(\varphi_{k}, \pi_{1}\left(A_{k}\right)\right)
$$

Podemos então reescrever a Proposição 2.1 ([15]) assim: 
Proposição 4.1. Qualquer classe de levantamento de $f_{k}: A_{k} \rightarrow A_{k}$ "pertence" a (ou está contida em) alguma classe de levantamento de $f: X \rightarrow X$, no sentido que $i_{k, F P C}\left[\tilde{f}_{k}\right]=[\tilde{f}]$, para algum $\tilde{f}$. Quando $\eta_{A_{k}} F i x\left(\tilde{f}_{k}\right)$ não é vazia, temos que: $\eta_{A_{k}} F i x\left(\tilde{f}_{k}\right)$ é um subconjunto de $\eta_{X} F i x(\tilde{f}) \Longleftrightarrow i_{k, F P C}\left[\tilde{f}_{k}\right]=[\tilde{f}]$.

Demonstração: [15]

Considere agora a inclusão $i_{k}: A_{k} \rightarrow X$. Temos um homomorfismo induzido

$$
i_{k_{\#}}: \pi_{1}\left(A_{k}\right) \rightarrow \pi_{1}(X)
$$

Como existem bijeções entre $r(f)$ e $\mathcal{R}\left(\varphi, \pi_{1}\right)$, e entre $r\left(f_{k}\right)$ e $\mathcal{R}\left(\varphi_{k}, \pi_{1}\left(A_{k}\right)\right)$; isomorfismos entre $\pi_{1}\left(A_{k}\right) \cong \operatorname{Cov}\left(\eta_{A_{k}}\right)$ e entre $\pi_{1}(X) \cong \operatorname{Cov}\left(\eta_{X}\right)$, e o conjunto $r\left(f, f_{A}\right)=\left\{C_{f} \supset C_{f_{A}}\right\}$ é formado pelos levantamentos $\tilde{f}$ de $f$ que estão na imagem dos levantamentos $\tilde{f}_{k}$ de $f_{k}$, para algum $k$, através da correspondência:

$$
i_{k, F P C}\left[\tilde{f}_{k}\right]=[\tilde{f}]
$$

podemos considerar o seguinte diagrama e definir $\hat{i}_{k}$ como a aplicação que faz o 
diagrama

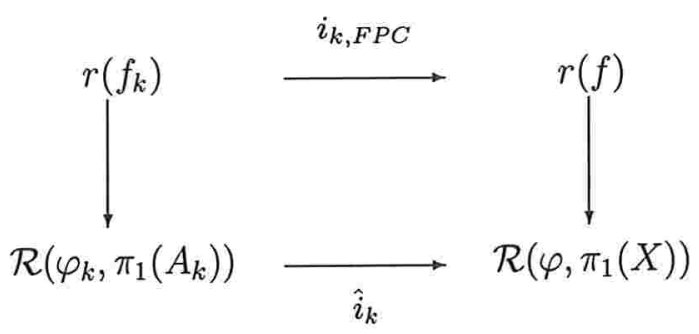

comutar, isto é:

$$
\hat{i}_{k}[\alpha]=\left[i_{k_{\#}} \alpha\right]
$$

Pode-se então definir (veja [3]) o seguinte conjunto:

$\mathcal{R}\left(\varphi, \varphi_{A}\right)=\left\{[\alpha] \in \mathcal{R}\left(\varphi, \pi_{1}(X)\right) \mid[\alpha]=\hat{i}_{k}[\beta]\right.$, para alguma $\left.[\beta] \in \mathcal{R}\left(\varphi_{k}, \pi_{1}\left(A_{k}\right)\right)\right\}$.

Assim, temos uma formulação algébrica para $r\left(f, f_{A}\right)$ : é o conjunto das classes de laços em $\pi_{1}(X)$ pela $\varphi$, que estão na imagem da classe de laços de $\pi_{1}(A)$ pela $\varphi_{A}$, através da aplicação $\hat{i}_{k}$, para algum $k$.

Deste modo, podemos reescrever o número de Reidemeister Relativo

$$
R(f ; X, A)=R(f)+R\left(f_{A}\right)-R\left(f, f_{A}\right)
$$

e o número de Reidemeister do Complemento

$$
R(f ; X-A)=R(f)-R\left(f, f_{A}\right)
$$


em função das classes de Reidemeister:

$$
\begin{aligned}
R(f ; X, A) & =\# \mathcal{R}\left(\varphi, \pi_{1}(X)\right)+\sum_{k} \# \mathcal{R}\left(\varphi_{k}, \pi_{1}\left(A_{k}\right)\right)-\# \mathcal{R}\left(\varphi, \varphi_{A}\right) \\
R(f ; X-A) & =\# \mathcal{R}\left(\varphi, \pi_{1}(X)\right)-\# \mathcal{R}\left(\varphi, \varphi_{A}\right) .
\end{aligned}
$$

Consideremos agora a tríade $\left(X, A_{1}, A_{2}\right)$ e $f:\left(X, A_{1}, A_{2}\right) \rightarrow\left(X, A_{1}, A_{2}\right)$ a aplicação da tríade.

Em vista das considerações anteriores e pela definição de $R\left(f ; A_{1} \cup A_{2}\right)$, temos o seguinte resultado:

Proposição 4.2. Seja a aplicação da tríade $f:\left(X, A_{1}, A_{2}\right) \rightarrow\left(X, A_{1}, A_{2}\right)$. Então o número de Reidemeister da tríade tem a seguinte expressão em função das cardinalidades das classes de Reidemeister:

$$
\begin{aligned}
R\left(f ; A_{1} \cup A_{2}\right)= & \# \mathcal{R}\left(\varphi_{1}, \pi_{1}\left(A_{1}\right)\right)+\# \mathcal{R}\left(\varphi_{2}, \pi_{1}\left(A_{2}\right)\right)+ \\
& +\sum_{k} \# \mathcal{R}\left(\varphi_{k}, \pi_{1}\left(A_{k}\right)\right)-\# \mathcal{R}\left(\varphi_{1}, \varphi_{0}\right)-\# \mathcal{R}\left(\varphi_{2}, \varphi_{0}\right)
\end{aligned}
$$

Demonstração: Pelas considerações anteriores, temos:

$$
\begin{aligned}
& R\left(f_{1} ; A_{1}, A_{0}\right)=\# \mathcal{R}\left(\varphi_{1}, \pi_{1}\left(A_{1}\right)\right)+\sum_{k} \# \mathcal{R}\left(\varphi_{k}, \pi_{1}\left(A_{k}\right)\right)-\# \mathcal{R}\left(\varphi_{1}, \varphi_{0}\right) \\
& R\left(f_{2} ; A_{2}, A_{0}\right)=\# \mathcal{R}\left(\varphi_{2}, \pi_{1}\left(A_{2}\right)\right)+\sum_{k} \# \mathcal{R}\left(\varphi_{k}, \pi_{1}\left(A_{k}\right)\right)-\# \mathcal{R}\left(\varphi_{2}, \varphi_{0}\right)
\end{aligned}
$$




$$
R\left(f_{0}\right)=\sum_{k} \# \mathcal{R}\left(\varphi_{k}, \pi_{1}\left(A_{k}\right)\right)
$$

Assim,

$$
\begin{aligned}
R\left(f ; A_{1} \cup A_{2}\right)= & \# \mathcal{R}\left(\varphi_{1}, \pi_{1}\left(A_{1}\right)\right)+\# \mathcal{R}\left(\varphi_{2}, \pi_{1}\left(A_{2}\right)\right)+\sum_{k} \# \mathcal{R}\left(\varphi_{k}, \pi_{1}\left(A_{k}\right)\right) \\
& -\# \mathcal{R}\left(\varphi_{1}, \varphi_{0}\right)-\# \mathcal{R}\left(\varphi_{2}, \varphi_{0}\right) .
\end{aligned}
$$

\subsection{Formulação Algébrica para Espaços de Jiang}

Em [15] Zhao obteve fórmulas algébricas para $N(f ; X-A)$ e $N(f ; X, A)$ no caso de $X$ e $A_{k} \subset \hat{A}$ serem espaços de Jiang. Como o número de Reidemeister da tríade $R\left(f ; A_{1} \cup A_{2}\right)$ é definido em termos de $R\left(f_{i} ; A_{i}, A_{0}\right), i=1,2$, e $R\left(f_{0}\right)$, ou em termos de $R\left(f_{i} ; A_{i}-A_{0}\right), i=1,2$, e $R\left(f_{0}\right)$, e vale a igualdade $R\left(f_{i} ; A_{i}, A_{0}\right)=N\left(f_{i} ; A_{i}, A_{0}\right)$ ou $R\left(f_{i} ; A_{i}-A_{0}\right)=N\left(f_{i} ; A_{i}-A_{0}\right), i=1,2$, quando $L\left(f_{i}\right) \neq 0$, obtemos então uma outra formulação algébrica para $R\left(f ; A_{1} \cup A_{2}\right)$ fundamentada nas fórmulas obtidas por Zhao.

Seja $f:(X, A) \rightarrow(X, A)$ aplicação do par, onde $(X, A)$ é um par de poliedros compactos. Para cada componente $A_{k} \subset \hat{A}$, escolhemos um ponto base $a_{k} \in A_{k}$ e um ponto base $x_{0} \in X$. Como os pontos do revestimento universal de $A_{k}$ e $X$ 
estão em correspondência com as classes de caminhos em $A_{k}$ e $X$ que partem de $a_{k}$ e $x_{0}$, podemos considerar então os caminhos constantes:

$$
\tilde{a}_{k}=\left\langle e_{k}\right\rangle \in \tilde{A}_{k} \quad \text { e } \quad \tilde{x}_{0}=\langle e\rangle \in \tilde{X}
$$

Sejam $w_{k}$ o caminho em $A_{k}$, com $w_{k}(0)=a_{k}, w_{k}(1)=f_{k}\left(a_{k}\right)$, para cada $k$, e $w_{0}$ o caminho em $X$, com $w_{0}(0)=x_{0}, w_{0}(1)=f\left(x_{0}\right)$. Então existem levantamentos únicos $\tilde{f}_{k}$ e $\tilde{f}$ tais que:

$$
\tilde{f}_{k}\left(\left\langle e_{k}\right\rangle\right)=\left\langle w_{k}\right\rangle \in \tilde{A}_{k} \quad \text { e } \quad \tilde{f}=(\langle e\rangle)=\left\langle w_{0}\right\rangle \in \tilde{X}
$$

Escolhendo os levantamentos $\tilde{f}_{k}$ e $\tilde{f}$ como referenciais, denotemos por:

$\nabla\left(f_{k} ; a_{k}, w_{k}\right)$ : o conjunto das $f_{k, \pi^{-}}$classes de conjugação em $\pi_{1}\left(A_{k}, a_{k}\right)$.

$\nabla\left(f ; x_{0}, w_{0}\right)$ : o conjunto das $f_{\pi^{-}}$classes de conjugação em $\pi_{1}\left(X, x_{0}\right)$.

Temos então o seguinte Lema:

Lema 4.1. Existem correspondências injetoras

$$
\begin{aligned}
\rho_{k}: \nabla\left(f_{k} ; a_{k}, w_{k}\right) & \rightarrow F P C\left(f_{k}\right) \\
\rho: \nabla\left(f ; x_{0}, w_{0}\right) & \rightarrow F P C(f)
\end{aligned}
$$


definidas por

$$
\begin{aligned}
\rho_{k}([\alpha]) & =\left[\alpha \circ \tilde{f}_{k}\right] \\
\rho([\beta]) & =[\beta \circ \tilde{f}]
\end{aligned}
$$

Demonstração: [7].

Para cada $k$, em [15] Zhao definiu

$$
\gamma_{k, \pi}: \pi_{1}\left(A_{k}, a_{k}\right) \rightarrow \pi_{1}\left(X, x_{0}\right)
$$

por

$$
\gamma_{k, \pi}(\langle\alpha\rangle)=\left(\tilde{i}_{k, \pi}\langle\alpha\rangle\right)\left\langle\gamma_{k}\right\rangle
$$

onde

$$
\left\langle\gamma_{k}\right\rangle=\left\langle u_{k} w_{k}\left(f \circ u_{k}\right)^{-1} w_{0}^{-1}\right\rangle
$$

Então, $\gamma_{k, \pi}$ induz uma transformação

$$
\gamma_{k}: \nabla\left(f_{k} ; a_{k}, w_{k}\right) \rightarrow \nabla\left(f ; x_{0}, w_{0}\right)
$$

e $\gamma_{k}$ independe da escolha do caminho $u_{k}$, onde $u_{k}$ é um caminho de $x_{0}$ até $a_{k}$. 
Zhao considerou então o seguinte diagrama comutativo

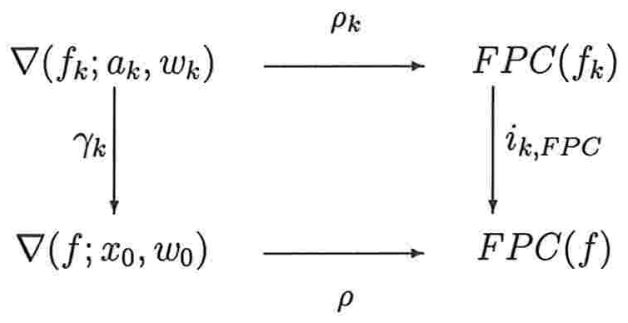

isto é:

$$
[\langle\alpha\rangle] \in \nabla\left(f_{k} ; a_{k}, w_{k}\right) \Longrightarrow i_{k, F P C} \circ \rho_{k}[\langle\alpha\rangle]=\rho \circ \gamma_{k}[\langle\alpha\rangle]
$$

ou seja:

$[\langle\alpha\rangle]$ se corresponde a uma classe de ponto fixo fracamente comum $\Longleftrightarrow$ está na imagem de algum $\gamma_{k}$ ([15], Teorema 4.4).

Para cada $k$, consideremos o seguinte diagrama comutativo

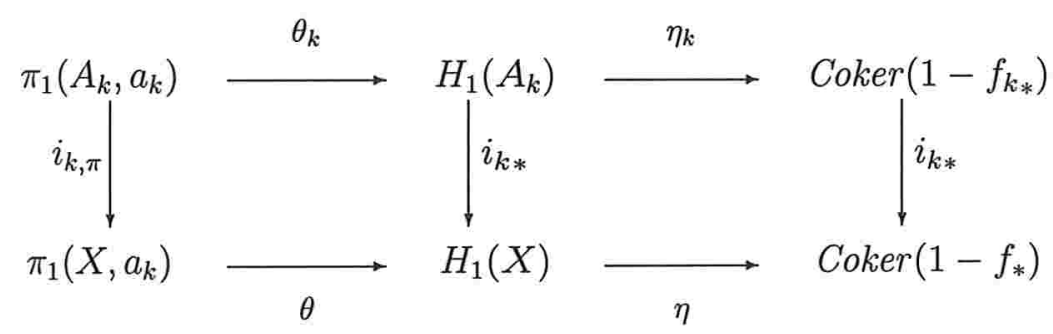

onde $\theta_{k}$ e $\theta$ são os abelianizadores, $\eta_{k}$ e $\eta$ as projeções naturais.

Pelo Lema 4.5 ([15]) o seguinte diagrama

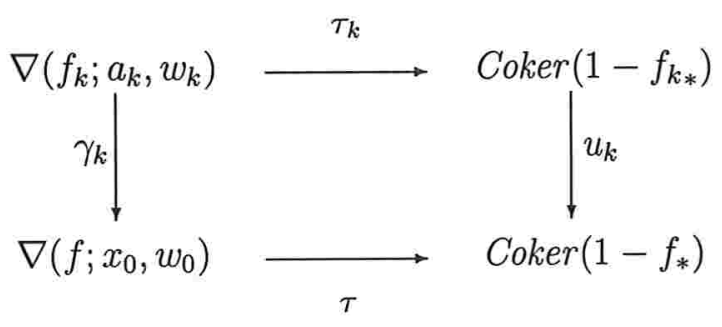


é comutativo, onde $\tau=\eta \circ \theta, \tau_{k}=\eta_{k} \circ \theta_{k}$ e $u_{k}(c)=i_{k *}(c)+\eta \circ \theta\left\langle\gamma_{k}\right\rangle$.

A seguir, usamos a formulação algébrica dos números $N(f ; X-A)$ e $N(f ; X, A)$ estabelecida por Zhao para caracterizar algebricamente o número de Reidemeister da tríade $R\left(f ; A_{1} \cup A_{2}\right)$.

Proposição 4.3. Seja $f:\left(X, A_{1}, A_{2}\right) \rightarrow\left(X, A_{1}, A_{2}\right)$ aplicação da tríade e suponhamos que $A_{1}$ e $A_{2}$ são espaços de Jiang, com $L\left(f_{i}\right) \neq 0, i=1,2, A_{0}=A_{1} \cap A_{2}$, $\hat{A}_{0}=\bigcup_{k=1}^{n} A_{0, k}$, onde $A_{0, k}$ é espaço de Jiang com $\prod_{k=1}^{m} L\left(f_{0, k}\right) \neq 0$ e $L\left(f_{0, q}\right)=0$, para $m<q \leq n$. Então

$$
\begin{aligned}
R\left(f ; A_{1} \cup A_{2}\right)= & \# \operatorname{Coker}\left(1-f_{1_{*}}\right)+\# \operatorname{Coker}\left(1-f_{2 *}\right)+ \\
& +\sum_{k=1}^{m} \operatorname{Coker}\left(1-f_{0, k_{*}}\right)-2 \#\left\{\bigcup_{k=1}^{m} u_{k} \operatorname{Coker}\left(1-f_{0, k_{*}}\right)\right\}
\end{aligned}
$$

Demonstração: Pelo Teorema 4.7 ([15]), para $i=1,2$, temos que

$$
\begin{aligned}
R\left(f_{i} ; A_{i}, A_{0}\right)=N\left(f_{i} ; A_{i}, A_{0}\right)= & \# \operatorname{Coker}\left(1-f_{i_{*}}\right)+\sum_{k=1}^{m} \# \operatorname{Coker}\left(1-f_{i, k_{*}}\right)- \\
& -\#\left\{\bigcup_{k=1}^{m} u_{k} \operatorname{Coker}\left(1-f_{i, k_{*}}\right)\right\},
\end{aligned}
$$

$\operatorname{mas} f_{i, k_{*}}=f_{0, k_{*}}$, para $i=1,2$ e temos que

$$
R\left(f_{0}\right)=N\left(f_{0}\right)=\sum_{k=1}^{m} N\left(f_{0, k}\right)=\sum_{k=1}^{m} \# \operatorname{Coker}\left(1-f_{0, k_{*}}\right)
$$




\section{Então:}

$$
\begin{aligned}
R\left(f ; A_{1}, A_{2}\right)= & \# \operatorname{Coker}\left(1-f_{1_{*}}\right)+\# \operatorname{Coker}\left(1-f_{2_{*}}\right)+ \\
& +\sum_{k=1}^{m} \# \operatorname{Coker}\left(1-f_{0, k_{*}}\right)-2 \#\left\{\bigcup_{k=1}^{m} u_{k} \operatorname{Coker}\left(1-f_{0, k_{*}}\right)\right\} .
\end{aligned}
$$




\section{Referências Bibliográficas}

[1] BROWN, R.F.; The Lefschetz Fixed Point Theorem, Scott, Foresman and Co., Glenview, IL, 1971.

[2] CARDONA, F.S.P.; Reidemeister Theory for Maps of Pairs, Doctoral Dissertation, UCLA, 1996.

[3] CARDONA, F.S.P.; WONG, P.N.S.; On the Computation of the Relative Nielsen Number, Topology Appl., vol.116 (2001), 29-41.

[4] CARDONA, F.S.P.; WONG, P.N.S.; The Relative Reidemeister Numbers of Fiber Map Pairs, Topological Methods in Nonlinear Analysis, Journal of the Juliusz Schauder Center, vol.21 (2003), 131-145.

[5] DOLD, A.; Lectures on Algebraic Topology, Springer Verlag, 1972.

[6] GONÇALVES, D.L.; KÜHL, J.C.S.; Teoria do Índice, $14^{\circ}$ Colóquio Brasileiro de Matemática, 1983. 
[7] JIANG, B.; Lectures on Nielsen Fixed Point Theory, Comtemporary Mathematics, vol.14, Amer. Math. Soc., Providence, RI, 1983.

[8] KIANG, T.; The Theory of Fixed Point Classes, Springer Verlag (Science Press), 1980.

[9] LYRA, C.B.; Grupo Fundamental e Revestimentos, IME-USP, São Paulo: 1969.

[10] MASSEY, W.S.; Algebraic Topology: An Introduction, Graduate Texts in Mathematic, vol.56, Springer Verlag, 1967.

[11] SCHIRMER, H.; A Relative Nielsen Number, Pacific J. Math. 122 (1986), pp. $459-473$.

[12] SCHIRMER, H.; Nielsen Numbers for Maps of Triades, Topology and its Applications, vol.52, North-Holland, (1993), 181-202.

[13] SCHIRMER, H.; A Survey of Relative Nielsen Fixed Point Theory, Contemp. Math. 152 (1993), pp. 291-309.

[14] VICK, J.W.; Homology Theory - An introduction to Algebraic Topology, Academic Press, New York: 1973. 
[15] ZHAO, X. Z.; A Relative Nielsen Number for the Complement, Topological Fixed Point Theory and its Applications (Proceedings. Tianjin 1988), Lectures Notes in Math, vol. 1411, Springer Verlag, 1989, pp. 189-199. 